Article

\title{
Diversity, Distribution, and Habitat Occurrence of the Diaptomid Copepods (Crustacea: Copepoda: Diaptomidae) in Freshwater Ecosystems of Thailand
}

\author{
Laorsri Sanoamuang $1,2, *(\mathbb{D})$ and Prapatsorn Dabseepai ${ }^{1}$ \\ 1 Applied Taxonomic Research Center, Faculty of Science, Khon Kaen University, Khon Kaen 40002, Thailand; \\ prapda@kku.ac.th \\ 2 Department of Biodiversity and Environmental Management, International College, Khon Kaen University, \\ Khon Kaen 40002, Thailand \\ * Correspondence: la_orsri@kku.ac.th
}

check for updates

Citation: Sanoamuang, L.; Dabseepai, P. Diversity, Distribution, and Habitat Occurrence of the Diaptomid Copepods (Crustacea:

Copepoda: Diaptomidae) in Freshwater Ecosystems of Thailand. Water 2021, 13, 2381. https:// doi.org/10.3390/w13172381

Academic Editors: Kay Van Damme and Alexey A. Kotov

Received: 13 July 2021

Accepted: 23 August 2021

Published: 30 August 2021

Publisher's Note: MDPI stays neutral with regard to jurisdictional claims in published maps and institutional affiliations.

Copyright: (c) 2021 by the authors. Licensee MDPI, Basel, Switzerland. This article is an open access article distributed under the terms and conditions of the Creative Commons Attribution (CC BY) license (https:// creativecommons.org/licenses/by/ $4.0 /)$.

\begin{abstract}
The diversity, distribution, and checklist of diaptomid copepods from various freshwater ecosystems throughout Thailand are presented, based on data from our biodiversity projects during 1993-2019 and literature reviews. Thailand has one of the most diversified diaptomid fauna in the world, with 42 species identified from 2150 localities (4962 samples). Mongolodiaptomus has the highest species richness with ten species, followed by Tropodiaptomus with seven species. Among these, eight taxa (Arctodiaptomus sp., Mongolodiaptomus pectinidactylus, Neodiaptomus meggitti, Tropodiaptomus hebereri, T. lanaonus, T. oryzanus, T. ruttneri, and Paradiaptomus greeni) are new to the fauna of Thailand. The rare P. greeni, which predominantly occurs in Africa, is also a new record for Southeast Asia. The most frequently encountered species were Mongolodiaptomus botulifer, Phyllodiaptomus praedictus, M. calcarus, M. dumonti, M. malaindosinensis, Vietodiaptomus blachei, Phyllodiaptomus christineae, Eodiaptomus sanoamuangae, Neodiaptomus yangtsekiangensis, E. draconisignivomi, T. vicinus, and Heliodiaptomus elegans. Twelve species appear to be endemic to Thailand, and eight species occur only in the countries belonging to the lower Mekong River Basin (Thailand, Cambodia, Laos, and Vietnam). The uniqueness of the Thai diaptomids is a high degree of co-occurrence of four to seven species in the same localities. Comments on the taxonomic status of the species recorded are provided. In addition, the taxonomic validity of Mongolodiaptomus malaindosinensis is discussed.
\end{abstract}

Keywords: Arctodiaptomus sp.; biodiversity; Calanoida; lower Mekong River Basin; Mongolodiaptomus malaindosinensis; Paradiaptomus greeni; Southeast Asia

\section{Introduction}

Copepods are among the most diverse micro-crustaceans in aquatic habitats [1]. They are abundant in freshwater ecosystems and can be a major component of most planktonic, benthic, and groundwater communities. Members of the family Diaptomidae in the order Calanoida are particularly successful in all kinds of freshwater habitats, comprising over 440 species [2]. Of the 22 genera ( 92 species) of freshwater diaptomids reported from inland waters of the Oriental biogeographic region, 10 genera (81 species) are endemic to this region [3].

Studies of the freshwater diaptomid copepods in Southeast Asia prior to 1980 were fragmentary. Of the 30 calanoid species, 27 diaptomid species were reported from Southeast Asia [3]. Most of the studies were conducted in Thailand since 1994 [4-22], while a few investigations have been documented in Cambodia [20,21,23], Laos [16,20,24], Malaysia [25-27], Singapore [28], the Philippines [29,30], and Indonesia [31]. A few studies were conducted in Vietnam [20], but most of the publications were published in Vietnamese with an English abstract [32]. 
In Thailand, Bricker et al. (1978) reported 7 diaptomid species from 11 reservoirs and one natural swampy lake in Thailand [33]. Later, a list of 14 species based on extensive collections sampled throughout the country was documented by Lai and Fernando (1981) [34]. In 1984, Boonsom (1984) examined samples from 39 different kinds of habitats, including rivers, reservoirs, irrigation tanks, ponds, and fish fields, revealing 8 diaptomid species [35]. In 1994-1998, four new species were described based on specimens from Thailand [4-7]. At the same time, Ranga Reddy et al. (1998) [7] published a list of 17 valid species for the country, and 16 of these were reported from the northeast region by Sanoamuang (1999) [9] as well. The misidentifications and taxonomic confusions of two species (Neodiaptomus mephistopheles Brehm, 1933 and Arctodiaptomus bacillifer (Koelbel, 1885)) [3,33,34,36] by previous researchers and the doubtful records of two taxa, Tropodiaptomus doriai (Richard, 1894) and Sinodiaptomus chaffanjoni (Richard, 1897), were corrected by Ranga Reddy et al. (1998) [7].

Consequently, there has been a gradual increase in our knowledge of the diversity of diaptomid copepods in Thailand. Since 2001, 13 more new species have been added to the list of Thailand [10-22]. As part of the authors' long-term intensive collections of freshwater copepods from different habitats all over Thailand during 1993-2019 and from the literature review, an updated list of diaptomid copepods known to date for the country is presented in this contribution. Their distributions within the six regions of Thailand, habitat occurrence, and taxonomic status of the diaptomid taxa are presented here.

\section{Materials and Methods}

\subsection{Study Area}

Thailand is situated in the heart of the Southeast Asian mainland, covering an area of 513,115 square kilometers. It lies in the humid tropics, having a monsoon climate with three distinct seasons-hot (March to April), rainy or monsoon (May to October), and cool (November to February). The country is officially divided into six geographical regions (Figure 1A) [36]. The northern region (with 9 provinces) is mountainous and was traditionally the most heavily forested area of the country. There are four main river basins (Ping, Wang, Yom, and Nan) in this region (Figure 1B). The northeast region (with 20 provinces) is some $200-300 \mathrm{~m}$ above sea level. This region is hemmed in by mountain ridges on the west and the south. This area is drained by the Song Khram, Chi, and Mun rivers, which are tributaries of the Mekong (Figure 1B). The central region (with 22 provinces) comprises the basins of the Chao Phraya and Pa Sak Rivers, which run from north to south and drain into the Gulf of Thailand. The western region (with 5 provinces) is also mountainous, alternating with plains along narrow valleys. The eastern region (with 7 provinces) is characterized by short mountain ranges and fruit plantations are a major component of agriculture in this area. The southern region (with 14 provinces) has the highest amount of rainfall in the country. It is the principal rubber-growing area and contains extensive alluvial deposits of tin.

\subsection{Sample Collections and Identifications}

Qualitative copepod samples of the pelagic and littoral zones from different freshwater habitats in Thailand were collected during 1993-2019. Information on 18 biodiversity projects in the surveyed water bodies is given in Table 1. A total of 4962 samples were collected from 2150 localities in 6 geographical regions (north, northeast, central, east, west, and south) of Thailand using a plankton net with a mesh size of $60 \mu \mathrm{m}$. In each site, sampling was performed either in two (dry and rainy) or three (summer, rainy, and win-ter) seasons, although some were carried out only once. A variety of water bodies, in-cluding permanent water habitats—canals, lakes, ponds, swamps, reservoirs, riversand temporary water habitats-rice fields, roadside canals, and temporary ponds- were sampled. The animals were preserved on-site in $4 \%$ formaldehyde or $70 \%$ ethanol. Adult copepods were selected, dissected, and prepared on a glycerin-mounted slide under an 
Olympus SZ51 stereomicroscope at 40-100- $\times$ magnification. All un-dissected specimens were stored in $70 \%$ ethanol in $1.5 \mathrm{~mL}$ microtubes.

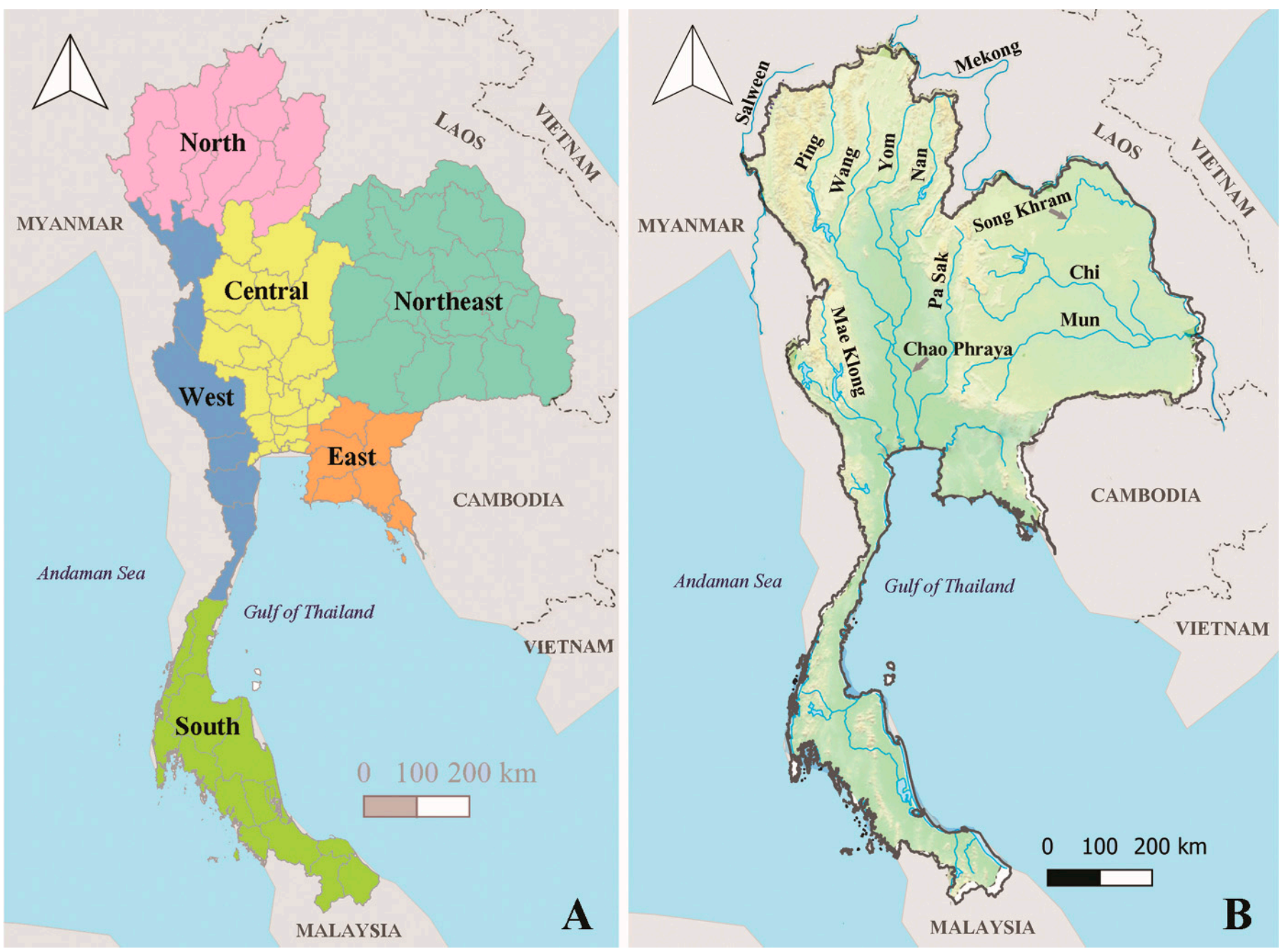

Figure 1. The map of Thailand displays six geographical regions (A) and major rivers (B).

Table 1. Information on sampling projects of the surveyed waterbodies in Thailand. The regions in Thailand are given by; $\mathrm{N}=$ North, $\mathrm{NE}=$ Northeast, $\mathrm{C}=$ Central, $\mathrm{E}=$ East, $\mathrm{W}=$ West, and $\mathrm{S}=$ South .

\begin{tabular}{|c|c|c|c|c|c|}
\hline No. & Sampling Habitats & $\begin{array}{l}\text { Regions in } \\
\text { Thailand }\end{array}$ & Sampling Years & $\begin{array}{l}\text { Number of } \\
\text { Localities }\end{array}$ & $\begin{array}{l}\text { Number of } \\
\text { Samples }\end{array}$ \\
\hline 1 & Various habitats in Northeast Thailand & $\mathrm{NE}$ & 1993 & 93 & 200 \\
\hline 2 & $\begin{array}{c}\text { Various habitats in Nakhon Ratchasima } \\
\text { and Surin Provinces }\end{array}$ & NE & 1996-1999 & 138 & 412 \\
\hline 3 & $\begin{array}{l}\text { Man-made reservoirs in the Phuphan } \\
\text { National Park }\end{array}$ & NE & 1997-1998 & 16 & 336 \\
\hline 4 & Major rivers in Thailand & $\mathrm{N}, \mathrm{NE}, \mathrm{C}, \mathrm{E}, \mathrm{W}, \mathrm{S}$ & 1998-1999 & 20 & 60 \\
\hline 5 & Various habitats throughout Thailand & $\mathrm{N}, \mathrm{NE}, \mathrm{C}, \mathrm{E}, \mathrm{W}, \mathrm{S}$ & 1998-2004 & 294 & 508 \\
\hline 6 & $\begin{array}{l}\text { Temporary-water habitats in seven } \\
\text { provinces of NE Thailand }\end{array}$ & NE & 1999-2003 & 521 & 844 \\
\hline 7 & $\begin{array}{l}\text { Various habitats in the floodplain of the } \\
\text { Rivers Nan, Chi, Mun and Song Khram }\end{array}$ & N,NE,C & 2000-2004 & 186 & 372 \\
\hline
\end{tabular}


Table 1. Cont.

\begin{tabular}{|c|c|c|c|c|c|}
\hline No. & Sampling Habitats & $\begin{array}{l}\text { Regions in } \\
\text { Thailand }\end{array}$ & Sampling Years & $\begin{array}{l}\text { Number of } \\
\text { Localities }\end{array}$ & $\begin{array}{l}\text { Number of } \\
\text { Samples }\end{array}$ \\
\hline 8 & $\begin{array}{l}\text { Various habitats in Central and } \\
\text { Northern Thailand }\end{array}$ & $\mathrm{N}, \mathrm{C}$ & 2001-2002 & 120 & 360 \\
\hline 9 & $\begin{array}{l}\text { Various habitats in Central and } \\
\text { Northern Thailand }\end{array}$ & $\mathrm{N}, \mathrm{C}$ & 2003 & 55 & 110 \\
\hline 10 & $\begin{array}{c}\text { Three natural lakes (Buemg Boraphet, } \\
\text { Bueng Khong Long, and Lake } \\
\text { Kud-Thing) }\end{array}$ & $\mathrm{NE}, \mathrm{C}$ & $2002-2004$ & 30 & 60 \\
\hline 11 & Various habitats in Eastern Thailand & $\mathrm{E}$ & $2003-2004$ & 55 & 110 \\
\hline 12 & Various habitats in Sisaket Province & NE & 2006-2007 & 99 & 190 \\
\hline 13 & $\begin{array}{l}\text { Various habitats in Suphan Buri, } \\
\text { Kanchanaburi, Ratchaburi and } \\
\text { Phetchaburi Provinces }\end{array}$ & $\mathrm{W}$ & 2007 & 200 & 218 \\
\hline 14 & $\begin{array}{l}\text { Various habitats in Nam Nao National } \\
\text { Park, Phetchabun Province }\end{array}$ & $\mathrm{C}$ & 2007-2009 & 10 & 75 \\
\hline 15 & $\begin{array}{c}\text { Lake Kud-Thing and various habitats } \\
\text { in Bueng Kan and Udon Thani } \\
\text { Provinces }\end{array}$ & NE & 2012-2014 & 97 & 408 \\
\hline 16 & $\begin{array}{l}\text { Various habitats in Chiang Rai, Phayao } \\
\text { and Nan Provinces }\end{array}$ & $\mathrm{N}$ & 2014-2016 & 67 & 154 \\
\hline 17 & $\begin{array}{l}\text { Various habitats in Khon Kaen, Kalasin } \\
\text { and Buriram Provinces }\end{array}$ & NE & 2014-2018 & 128 & 344 \\
\hline 18 & $\begin{array}{c}\text { Various habitats in Saraburi and } \\
\text { Lopburi Provinces }\end{array}$ & C & 2019 & 21 & 201 \\
\hline \multicolumn{4}{|c|}{ Total } & 2150 & 4962 \\
\hline
\end{tabular}

All appendages and body ornamentation were examined at $1000-\times$ magnification under an Olympus CX31 compound microscope. Specimens for scanning electron microscopy (SEM) were dehydrated in an ethanol series (50,70, 80, 90, 95, 100, and 100\%) for $15 \mathrm{~min}$ at each concentration. Specimens were dried in a critical-point dryer and were mounted on stubs using adhesive tape under a stereomicroscope. Dried specimens were coated with gold in a sputter coater. The SEM photographs were taken using a scanning electron microscope (LEO, 1450VP).

The abbreviations used in this paper are as follows:

Exp-2 = second exopodal segment;

P5 $=$ leg 5.

\subsection{Data Analysis}

The relative occurrences of the diaptomid copepods recorded from Thailand are cat-egorized according to the occurrence frequencies of the local populations into five catego-ries as follows: (1) common $=$ species that occurs in more than $45 \%$ of the sampled locali-ties; (2) fairly common = species that occurs in $31-45 \%$ of the sampled localities; (3) un-common $=$ species that occurs in $16-30 \%$ of the sampled localities; $(4)$ rare $=$ species that occurs in $5-15 \%$ of the sampled localities; (5) extremely rare = species that occurs in less than $5 \%$ of the sampled localities.

\section{Results}

\subsection{Species Diversity}

A list of diaptomid species identified from the samples examined, along with their habitat types, relative occurrences, and distributional regions in Thailand, is provided in 
Table 2. Of the 42 species identified, 41 species are members of the subfamily Diaptominae, while only 1 belongs to the subfamily Paradiaptominae. The diversity of species among the 11 genera of the Diaptomidae is dominated by the genus Mongolodiaptomus, with 10 known species, followed by Tropodiaptomus (7 species), Neodiaptomus (6 species) and Phyllodiaptomus ( 6 species). Among these, 17 species ( $40.5 \%$ of the species recorded) have been originally described from specimens from Thailand since 1994, including (1) Phyllodiaptomus (Ctenodiaptomus) praedictus Dumont and Ranga Reddy, 1994 (Figure 2A); (2) Phyllodiaptomus (Phyllodiaptomus) christineae Dumont, Ranga Reddy, and Sanoamuang, 1996 (Figure 2B); (3) Eodiaptomus sanoamuangae Ranga Reddy and Dumont, 1998 (Figure 3A); (4) Mongolodiaptomus rarus (Ranga Reddy, Sanoamuang, and Dumont, 1998); (5) Phyllodiaptomus (Ctenodiaptomus) surinensis Sanoamuang and Yindee, 2001; (6) Eodiaptomus phuphanensis Sanoamuang, 2001 (Figure 3B); (7) Mongolodiaptomus dumonti Sanoamuang, 2001 (Figure 4A); (8) Neodiaptomus songkhramensis Sanoamuang and Athi-bai, 2002; (9) Heliodiaptomus phuthaiorum Sanoamuang, 2004 (Figure 5A); (10) Eodiaptomus phuvongi Sanoamuang and Sivongxay, 2005 (Figure 3C); (11) Phyllodiaptomus (Phyllodiaptomus) thailandicus Sanoamuang and Teeramaethee, 2006 (Figure 2C); (12) Neodiaptomus siamensis Proogkiat and Sanoamuang, 2008; (13) Mongolodiaptomus loeiensis Watiroyram and Sanoamuang, 2017 (Figure 4B); (14) Mongolodiaptomus mekongensis Sanoamuang and Watiroyram, 2018 (Figure 4C); (15) Phyllodiaptomus (Phyllodiaptomus) roietensis (Sanoamuang and Watiroyram, 2020) (Figure 2D); (16) Tropodiaptomus megahyaline Sae-tang, Sanoamuang, and Maiphae, 2020; (17) Dentodiaptomus orientalis Sanoamuang and Watiroyram, 2021. In addition to the above, four more undescribed species (Arctodiaptomus sp., Mongolodiaptomus sp., Phyllodiaptomus sp. and Tropodiaptomus sp.) are waiting to be described.

Eight taxa-namely, Arctodiaptomus sp. (Figure 5B), Mongolodiaptomus pectinidactylus (Shen and Tai, 1964) (Figure 4D), Neodiaptomus meggitti Kiefer, 1932, Tropodiaptomus hebereri (Kiefer, 1930) (Figure 6A), T. lanaonus Kiefer, 1982, T. oryzanus Kiefer, 1937 (Figure 6B,C), T. ruttneri (Brehm, 1923) (Figure 6D), and Paradiaptomus greeni (Gurney, 1906) (Figure 5C,D) are new to the fauna of Thailand. Moreover, this is the first record of a species complex of $P$. greeni in the subfamily Paradiaptominae in Southeast Asia.

\subsection{Relative Occurrences and Distribution}

Twelve species (28.6\%) are commonly found in different kinds of habitats, and most are widely distributed throughout the six regions of Thailand. The most frequently encountered species in the sampled localities were Mongolodiaptomus botulifer (Figure 7A), Phyllodiaptomus praedictus (Figure 2A), M. calcarus (Figure 7B), M. dumonti (Figure 4A), M. malaindosinensis (Figure 7C), Vietodiaptomus blachei (Figure 8A), P. christineae (Figure 2B), Eodiaptomus sanoamuangae (Figure 3A), Neodiaptomus yangtsekiangensis, E. draconisignivomi (Figure 3D), T. vicinus (Figure 6E), and H. elegans (Figure 8B). The rest, including five fairly common, nine uncommon, seven rare, and nine extremely rare species, are also listed in Table 2.

The species richness of the diaptomids ranged from 1 to 7 species per locality at the same sampling dates. Although $2-3$ species usually coexist in the same location, the co-existence of 4-7 diaptomid species has been observed in northeastern Thailand, particularly in temporary water habitats (Table 3 ). The co-occurrence of congeneric species was about $50 \%$ of the localities having co-occurrence records, and it was predominantly found in small water bodies. M. botulifer showed the highest co-occurrences with other species, followed by M. dumonti, M. malaindosinensis, P. (P.) christineae, and V. blachei. 


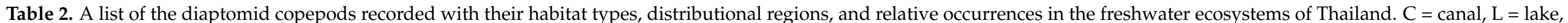

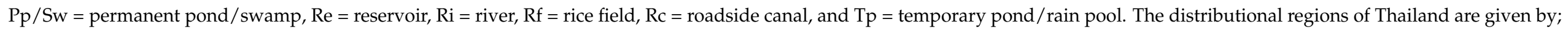

$\mathrm{N}=$ North, $\mathrm{NE}=$ Northeast, $\mathrm{C}=$ Central, $\mathrm{E}=$ East, $\mathrm{W}=$ West, and $\mathrm{S}=$ South. Species with bold letters and marked with * are new records for Thailand.

\begin{tabular}{|c|c|c|c|c|c|c|c|c|c|c|c|c|c|c|c|c|}
\hline \multirow{3}{*}{ No } & \multirow{3}{*}{ Species } & \multicolumn{8}{|c|}{ Habitat Types } & \multicolumn{6}{|c|}{ Distributional Regions } & \multirow{3}{*}{$\begin{array}{c}\text { Relative } \\
\text { Occurrences }\end{array}$} \\
\hline & & \multirow[b]{2}{*}{$\mathrm{C}$} & \multicolumn{4}{|c|}{ Permanent Waters } & \multicolumn{3}{|c|}{ Temporary Waters } & \multirow[b]{2}{*}{$\mathbf{N}$} & \multirow[b]{2}{*}{ NE } & \multirow[b]{2}{*}{$\mathrm{C}$} & \multirow[b]{2}{*}{$\mathrm{E}$} & \multirow[b]{2}{*}{$\mathbf{W}$} & \multirow[b]{2}{*}{$\mathbf{S}$} & \\
\hline & & & $\mathbf{L}$ & $\mathrm{Pp} / \mathrm{Sw}$ & $\operatorname{Re}$ & $\mathbf{R i}$ & $\mathbf{R f}$ & Rc & Tp & & & & & & & \\
\hline 1 & Allodiaptomus raoi Kiefer, 1936 & & $\checkmark$ & & $\boldsymbol{V}$ & $\boldsymbol{V}$ & $\checkmark$ & & & & $\checkmark$ & & & & & uncommon \\
\hline 2 & Arctodiaptomus sp. & & & & & $\boldsymbol{V}$ & & & & & $\boldsymbol{v}$ & & & & & extremely rare \\
\hline 3 & Dentodiaptomus javanus (Grochmalicki, 1915) & & & $\checkmark$ & & & $\boldsymbol{V}$ & $\checkmark$ & $\boldsymbol{\nu}$ & $\boldsymbol{\nu}$ & $\checkmark$ & & $\checkmark$ & $\boldsymbol{\nu}$ & & fairly common \\
\hline 4 & $\begin{array}{c}\text { Dentodiaptomus orientalis Sanoamuang \& } \\
\text { Watiroyram, } 2021\end{array}$ & & & $\checkmark$ & & & & $\boldsymbol{v}$ & $\boldsymbol{v}$ & & $\boldsymbol{v}$ & & & & & uncommon \\
\hline 5 & Eodiaptomus draconisignivomi Brehm, 1952 & $\checkmark$ & $\checkmark$ & $\checkmark$ & $\boldsymbol{\nu}$ & $\checkmark$ & & & & & $\checkmark$ & $\checkmark$ & $\checkmark$ & $\checkmark$ & & fairly common \\
\hline 6 & Eodiaptomus phuphanensis Sanoamuang, 2001 & & & & $\checkmark$ & $\checkmark$ & & $\checkmark$ & $\boldsymbol{V}$ & & $\checkmark$ & & & $\checkmark$ & & fairly common \\
\hline 7 & $\begin{array}{l}\text { Eodiaptomus phuvongi Sanoamuang \& Sivongxay, } \\
\qquad 2004\end{array}$ & $\boldsymbol{v}$ & & $\boldsymbol{v}$ & & & & & $\boldsymbol{\sim}$ & & $\boldsymbol{v}$ & & & & & fairly common \\
\hline 8 & $\begin{array}{c}\text { Eodiaptomus sanoamuangae Ranga Reddy \& } \\
\text { Dumont, } 1998\end{array}$ & $\boldsymbol{V}$ & $\boldsymbol{v}$ & $\checkmark$ & $\boldsymbol{v}$ & & $\boldsymbol{V}$ & $\checkmark$ & $\checkmark$ & $\boldsymbol{V}$ & $\boldsymbol{V}$ & $\boldsymbol{v}$ & & $\checkmark$ & & common \\
\hline 9 & Heliodiaptomus elegans Kiefer, 1935 & & $\checkmark$ & $\boldsymbol{r}$ & $\boldsymbol{V}$ & $\checkmark$ & & & $\boldsymbol{r}$ & $\checkmark$ & $\checkmark$ & & & $\boldsymbol{V}$ & & fairly common \\
\hline 10 & Heliodiaptomus phuthaiorum Sanoamuang, 2004 & & & & & & & $\checkmark$ & $\boldsymbol{\nu}$ & & $\boldsymbol{V}$ & & & & & rare \\
\hline 11 & Heliodiaptomus viduus (Gurney, 1916) & $\checkmark$ & $\checkmark$ & $\checkmark$ & $\boldsymbol{\sim}$ & & & & & $\boldsymbol{V}$ & & $\checkmark$ & $\checkmark$ & $\boldsymbol{\sim}$ & & uncommon \\
\hline 12 & Mongolodiaptomus botulifer (Kiefer, 1974) & $\checkmark$ & $\checkmark$ & $\checkmark$ & $\boldsymbol{\sim}$ & $\checkmark$ & $\checkmark$ & $\checkmark$ & $\checkmark$ & $\checkmark$ & $\boldsymbol{\nu}$ & $\checkmark$ & $\checkmark$ & $\checkmark$ & $\checkmark$ & common \\
\hline 13 & Mongolodiaptomus calcarus (Shen \& Tai, 1965) & $\checkmark$ & $\checkmark$ & $\checkmark$ & $\checkmark$ & $\checkmark$ & $\checkmark$ & $\checkmark$ & $\checkmark$ & $\checkmark$ & $\checkmark$ & $\checkmark$ & & $\checkmark$ & $\checkmark$ & common \\
\hline 14 & Mongolodiaptomus dumonti Sanoamuang, 2001 & $\checkmark$ & $\checkmark$ & $\checkmark$ & $\boldsymbol{r}$ & $\checkmark$ & $\checkmark$ & $\boldsymbol{r}$ & $\checkmark$ & $\boldsymbol{V}$ & $\boldsymbol{V}$ & $\checkmark$ & $\checkmark$ & $\boldsymbol{V}$ & & common \\
\hline 15 & $\begin{array}{c}\text { Mongolodiaptomus loeiensis Watiroyram \& } \\
\text { Sanoamuang, } 2017\end{array}$ & & & & & & & & $\checkmark$ & & $\boldsymbol{v}$ & & & & & extremely rare \\
\hline 16 & $\begin{array}{l}\text { Mongolodiaptomus malaindosinensis (Lai \& } \\
\text { Fernando, 1978) }\end{array}$ & & $\checkmark$ & $\boldsymbol{\checkmark}$ & $\checkmark$ & $\boldsymbol{v}$ & & & $\checkmark$ & $\checkmark$ & $\checkmark$ & $\checkmark$ & $\checkmark$ & & $\checkmark$ & common \\
\hline 17 & $\begin{array}{c}\text { Mongolodiaptomus mekongensis Sanoamuang \& } \\
\text { Watiroyram, } 2018\end{array}$ & & & $\checkmark$ & & & $\checkmark$ & $\checkmark$ & $\checkmark$ & & $\checkmark$ & & & & & uncommon \\
\hline
\end{tabular}


Table 2. Cont

\begin{tabular}{|c|c|c|c|c|c|c|c|c|c|c|c|c|c|c|c|c|}
\hline \multirow{3}{*}{ No } & \multirow{3}{*}{ Species } & \multicolumn{8}{|c|}{ Habitat Types } & \multicolumn{6}{|c|}{ Distributional Regions } & \multirow{3}{*}{$\begin{array}{c}\text { Relative } \\
\text { Occurrences }\end{array}$} \\
\hline & & \multirow[b]{2}{*}{$\mathrm{C}$} & \multicolumn{4}{|c|}{ Permanent Waters } & \multicolumn{3}{|c|}{ Temporary Waters } & \multirow[b]{2}{*}{$\mathbf{N}$} & \multirow[b]{2}{*}{ NE } & \multirow[b]{2}{*}{$\mathrm{C}$} & \multirow[b]{2}{*}{$\mathbf{E}$} & \multirow[b]{2}{*}{$\mathbf{W}$} & \multirow[b]{2}{*}{$\mathrm{S}$} & \\
\hline & & & $\mathbf{L}$ & $\mathrm{Pp} / \mathrm{Sw}$ & $\operatorname{Re}$ & $\mathbf{R i}$ & $\mathbf{R f}$ & Rc & $\mathrm{Tp}$ & & & & & & & \\
\hline 18 & $\begin{array}{l}\text { Mongolodiaptomus pectinidactylus (Shen \& Tai, } \\
\text { 1964) * }\end{array}$ & & $\boldsymbol{r}$ & & & & & & $\boldsymbol{v}$ & & $\checkmark$ & & & & & rare \\
\hline 19 & $\begin{array}{l}\text { Mongolodiaptomus rarus (Ranga Reddy, } \\
\text { Sanoamuang \& Dumont, 1998) }\end{array}$ & & & & & & $\checkmark$ & $\boldsymbol{v}$ & $\checkmark$ & & $\boldsymbol{V}$ & & & & & uncommon \\
\hline 20 & Mongolodiaptomus uenoi (Kikuchi, 1936) & $\checkmark$ & & $\checkmark$ & $\boldsymbol{\sim}$ & $\checkmark$ & & $\checkmark$ & $\boldsymbol{\nu}$ & & $\boldsymbol{\sim}$ & & & & & fairly common \\
\hline 21 & Mongolodiaptomus sp. & & & $\checkmark$ & & & & & $\checkmark$ & & $\boldsymbol{V}$ & & & & & extremely rare \\
\hline 22 & Neodiaptomus laii Kiefer, 1974 & $\checkmark$ & & $\checkmark$ & & & & & $\checkmark$ & & $\checkmark$ & & $\boldsymbol{\sim}$ & & $\boldsymbol{\sim}$ & uncommon \\
\hline 23 & Neodiaptomus meggitti Kiefer, 1932 * & & & $\checkmark$ & & & & & & $\checkmark$ & & & & & & extremely rare \\
\hline 24 & Neodiaptomus schmackeri (Poppe \& Richard, 1892) & $\boldsymbol{V}$ & & $\checkmark$ & & & $\boldsymbol{V}$ & $\checkmark$ & $\boldsymbol{V}$ & $\checkmark$ & & $\boldsymbol{V}$ & & $\checkmark$ & & fairly common \\
\hline 25 & $\begin{array}{l}\text { Neodiaptomus siamensis Proogkiat \& Sanoamuang, } \\
2008\end{array}$ & & & & & & & & $\boldsymbol{v}$ & & & $\checkmark$ & & & & extremely rare \\
\hline 26 & $\begin{array}{c}\text { Neodiaptomus songkhramensis Sanoamuang \& } \\
\text { Athibai, } 2002\end{array}$ & & & & & & $\checkmark$ & $\checkmark$ & $\checkmark$ & & $\boldsymbol{v}$ & & & & & uncommon \\
\hline 27 & Neodiaptomus yangtsekiangensis Mashiko, 1951 & $\checkmark$ & $\boldsymbol{\sim}$ & $\checkmark$ & $\boldsymbol{\sim}$ & $\checkmark$ & & $\checkmark$ & $\checkmark$ & & $\boldsymbol{\nu}$ & & $\checkmark$ & & $\checkmark$ & common \\
\hline 28 & $\begin{array}{l}\text { Phyllodiaptomus (Ctenodiaptomus) praedictus } \\
\text { Dumont \& Ranga Reddy, } 1994\end{array}$ & $\checkmark$ & $\checkmark$ & $\checkmark$ & $\boldsymbol{v}$ & $\checkmark$ & $\checkmark$ & $\checkmark$ & $\boldsymbol{v}$ & $\checkmark$ & $\boldsymbol{v}$ & $\checkmark$ & $\boldsymbol{V}$ & $\checkmark$ & & common \\
\hline 29 & $\begin{array}{l}\text { Phyllodiaptomus (Ctenodiaptomus) surinensis } \\
\text { Sanoamuang \& Yindee, } 2001\end{array}$ & $\checkmark$ & & & & & & & & & $\checkmark$ & & & & & extremely rare \\
\hline 30 & $\begin{array}{l}\text { Phyllodiaptomus (Phyllodiaptomus) christineae } \\
\text { Dumont, Ranga Reddy \& Sanoamuang, } 1996\end{array}$ & $\checkmark$ & $\checkmark$ & $\checkmark$ & $\boldsymbol{\nu}$ & $\checkmark$ & & & $\boldsymbol{\nu}$ & $\checkmark$ & $\boldsymbol{V}$ & $\checkmark$ & $\checkmark$ & $\checkmark$ & & common \\
\hline 31 & $\begin{array}{l}\text { Phyllodiaptomus (Phyllodiaptomus) roietensis } \\
\text { Sanoamuang \& Watiroyram, } 2020\end{array}$ & & & & & & $\boldsymbol{\nu}$ & $\checkmark$ & $\boldsymbol{\nu}$ & & $\boldsymbol{v}$ & & & & & rare \\
\hline 32 & $\begin{array}{l}\text { Phyllodiaptomus (Phyllodiaptomus) thailandicus } \\
\text { Sanoamuang \& Teeramaethee, } 2006\end{array}$ & $\checkmark$ & & $\checkmark$ & & & & & $\boldsymbol{v}$ & & & & $\checkmark$ & $\checkmark$ & $\checkmark$ & rare \\
\hline 33 & Phyllodiaptomus (Phyllodiaptomus) sp. & & & $\checkmark$ & & & & & $\boldsymbol{V}$ & & $\checkmark$ & & & & & rare \\
\hline 34 & Tropodiaptomus hebereri (Kiefer, 1930) * & $\checkmark$ & & & & & & & & $\checkmark$ & & & & & & extremely rare \\
\hline 35 & Tropodiaptomus lanaonus Kiefer, 1982 * & & $\checkmark$ & & & & & & & & $\checkmark$ & $\checkmark$ & & & & rare \\
\hline
\end{tabular}


Table 2. Cont

\begin{tabular}{|c|c|c|c|c|c|c|c|c|c|c|c|c|c|c|c|c|}
\hline \multirow{3}{*}{ No } & \multirow{3}{*}{ Species } & \multicolumn{8}{|c|}{ Habitat Types } & \multicolumn{6}{|c|}{ Distributional Regions } & \multirow{3}{*}{$\begin{array}{c}\text { Relative } \\
\text { Occurrences }\end{array}$} \\
\hline & & \multicolumn{5}{|c|}{ Permanent waters } & \multicolumn{9}{|c|}{ Temporary waters } & \\
\hline & & $\mathrm{C}$ & $\mathbf{L}$ & $\mathrm{Pp} / \mathrm{Sw}$ & $\operatorname{Re}$ & $\mathbf{R i}$ & Rf & Rc & $\mathrm{Tp}$ & $\mathbf{N}$ & NE & $\mathrm{C}$ & $\mathbf{E}$ & $\mathbf{W}$ & $\mathrm{S}$ & \\
\hline 37 & Tropodiaptomus oryzanus Kiefer, 1937 * & & $\checkmark$ & & & & $\checkmark$ & $\boldsymbol{v}$ & $\boldsymbol{v}$ & & $\boldsymbol{v}$ & & & & & uncommon \\
\hline 38 & Tropodiaptomus ruttneri $(\mathrm{Brehm}, 1923)$ * & & & & & & $\boldsymbol{v}$ & & $\boldsymbol{v}$ & $\checkmark$ & $\boldsymbol{v}$ & & & & & uncommon \\
\hline 40 & Tropodiaptomus sp. & & & & & & & & $\boldsymbol{V}$ & $\checkmark$ & & & & & & extremely rare \\
\hline \multirow[t]{2}{*}{41} & Vietodiaptomus blachei (Brehm, 1951) & $\checkmark$ & $\checkmark$ & $\checkmark$ & $\boldsymbol{V}$ & & $\checkmark$ & $\boldsymbol{v}$ & $\checkmark$ & $\checkmark$ & $\checkmark$ & $\checkmark$ & & $\boldsymbol{v}$ & $\checkmark$ & common \\
\hline & Subfamily Paradi & tomi & e Ki & r, 1932 & & & & & & & & & & & & \\
\hline \multirow[t]{2}{*}{42} & Paradiaptomus greeni (Gurney, 1906) * & & & & & & & & $\boldsymbol{v}$ & & $\boldsymbol{V}$ & & & & & extremely rare \\
\hline & & Tot & & & & & & & & 17 & 35 & 14 & 11 & 15 & 8 & \\
\hline
\end{tabular}



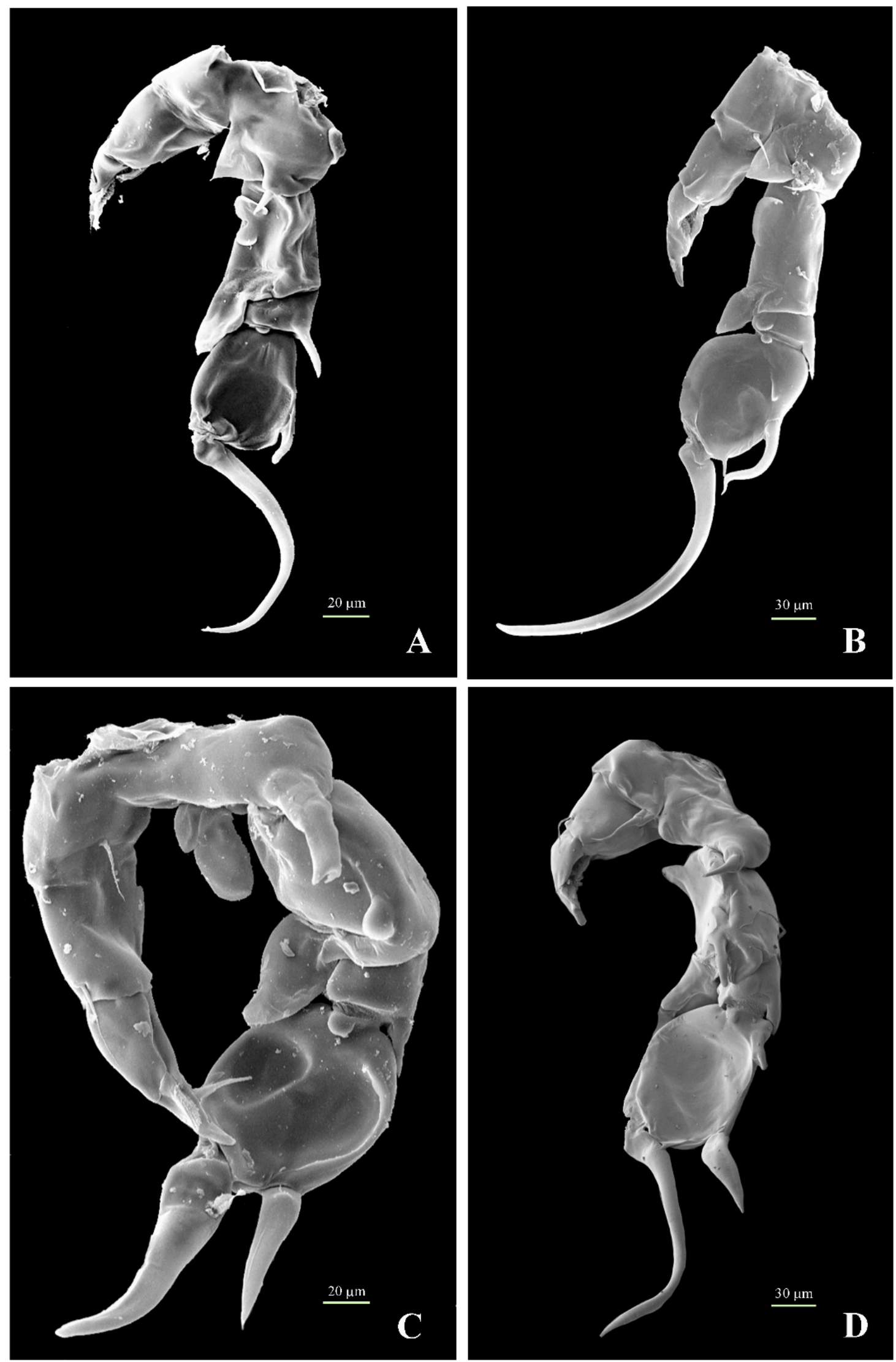

Figure 2. SEM photographs of the male leg 5 (P5) of four species of the genus Phyllodiaptomus: (A) P. (C.) praedictus; (B). P. (P.) christineae; (C). P. (P.) thailandicus; and (D). P. (P.) roietensis. 


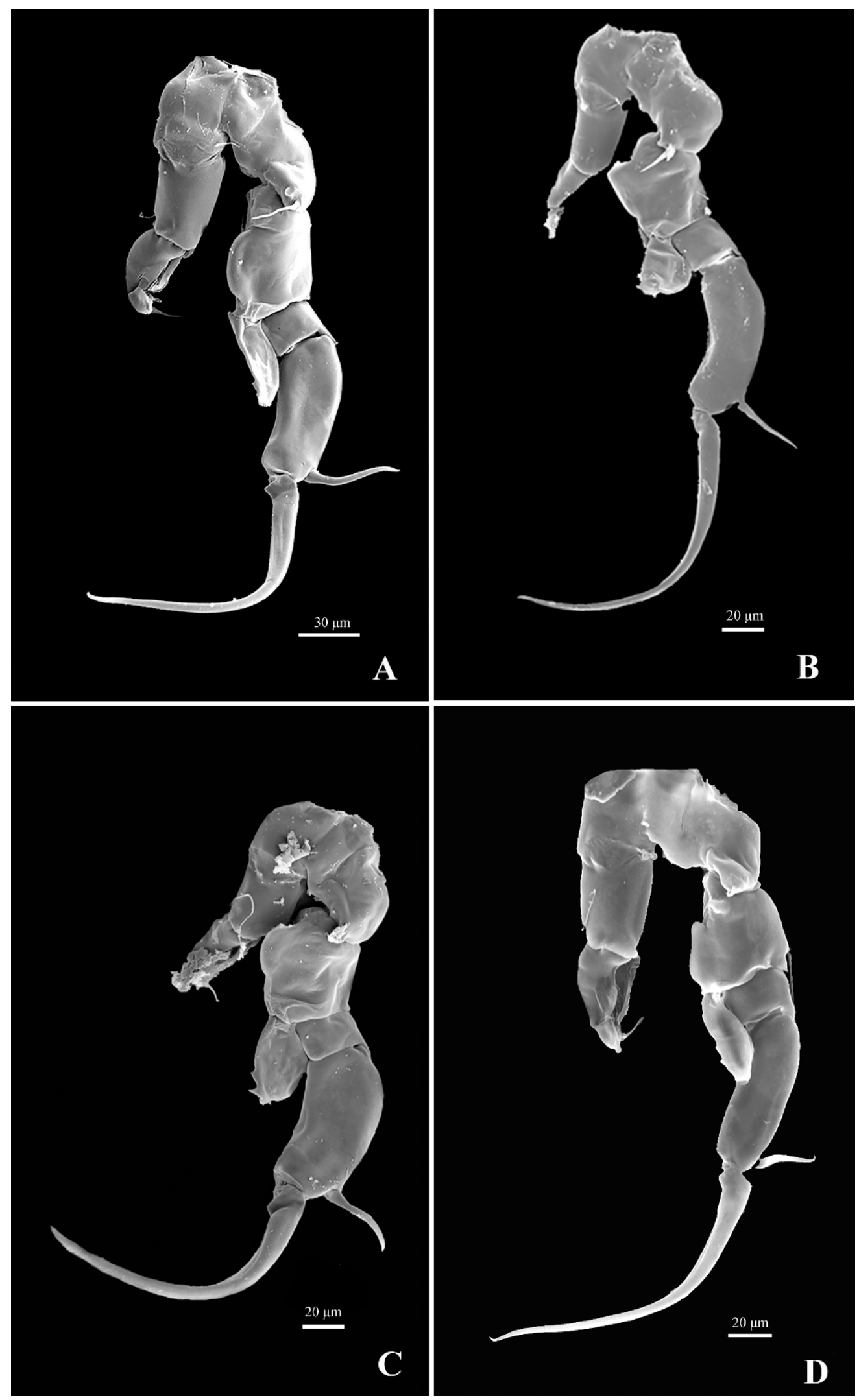

Figure 3. SEM photographs of the male leg 5 (P5) of four species of the genus Eodiaptomus: (A) E. sanoamuangae; (B) E. Phuphanensis; (C) E. phuvongi; and (D) E. draconisignivomi. 

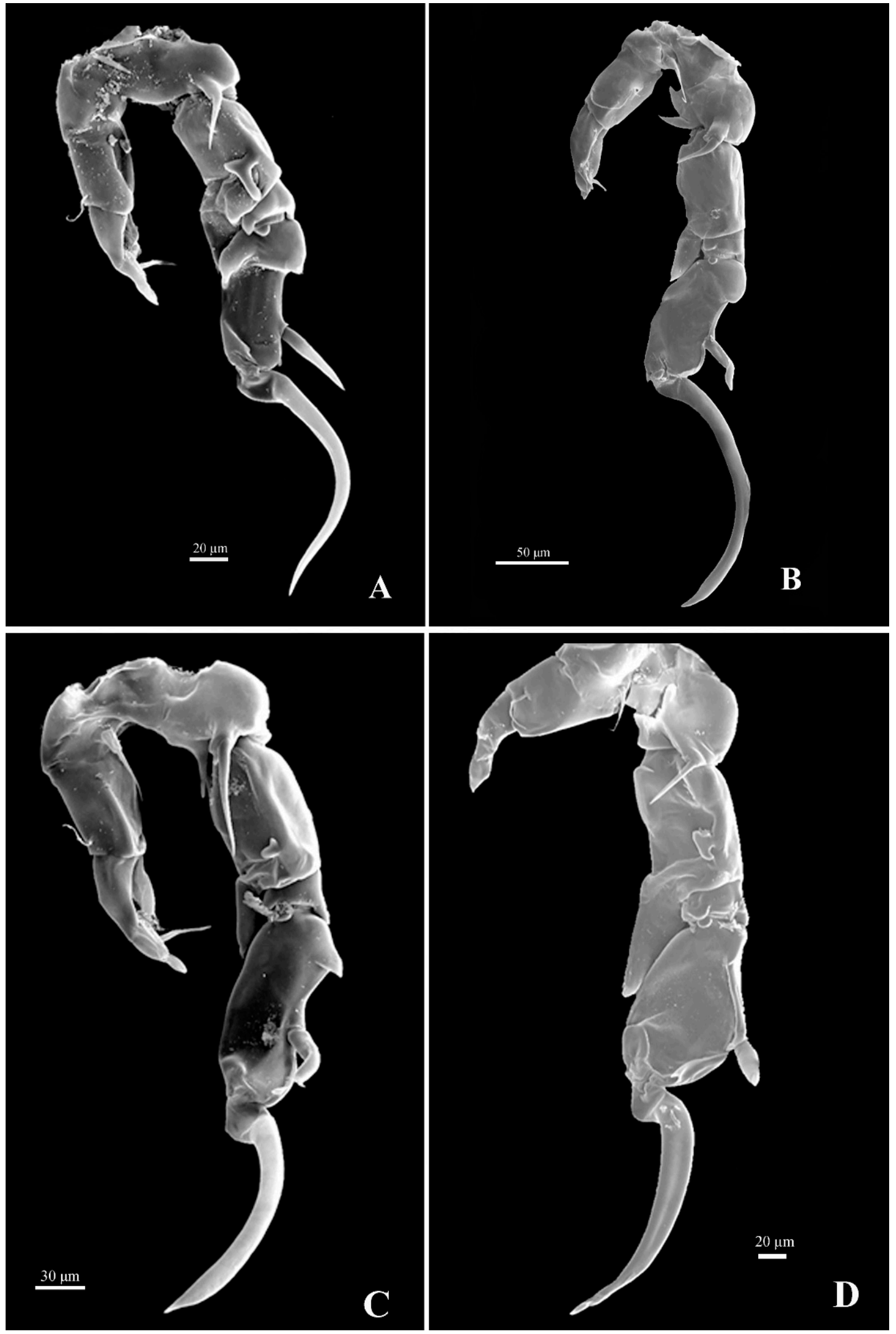

Figure 4. SEM photographs of the male leg 5 (P5) of four species of the genus Mongolodiaptomus: (A) M. dumonti; (B) M. loeiensis; (C) M. mekongensis; and (D) M. pectinidactylus. 

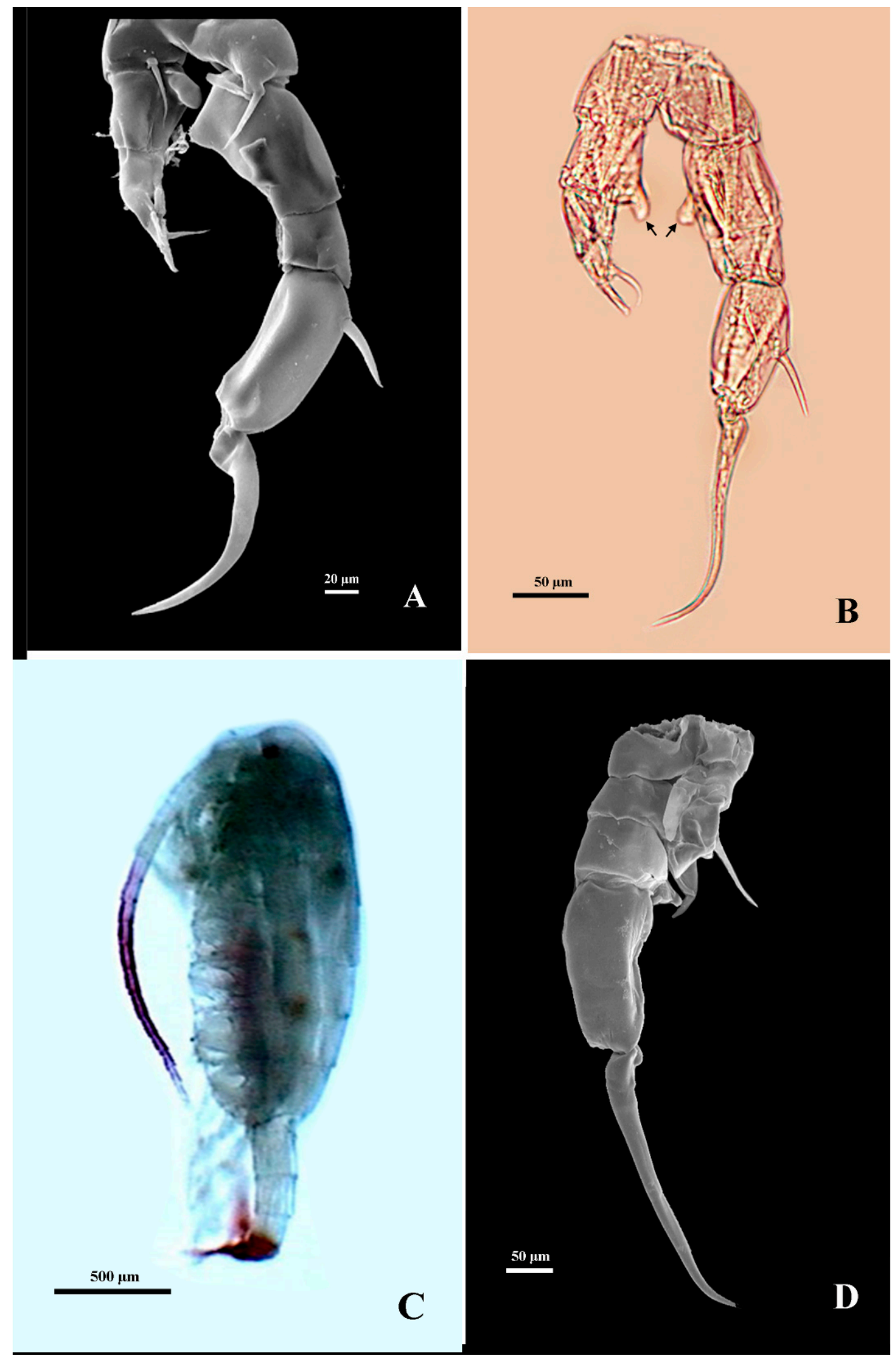

Figure 5. Photographs of three species of diaptomid copepods. (A) male P5 of Heliodiaptomus phuthaiorum; (B) male P5 of Arctodiaptomus sp. (black arrows point to left and right hyaline membranes on left and right bases; (C) habitus of male Paradiaptomus greeni; (D) male P5 of P. greeni. 

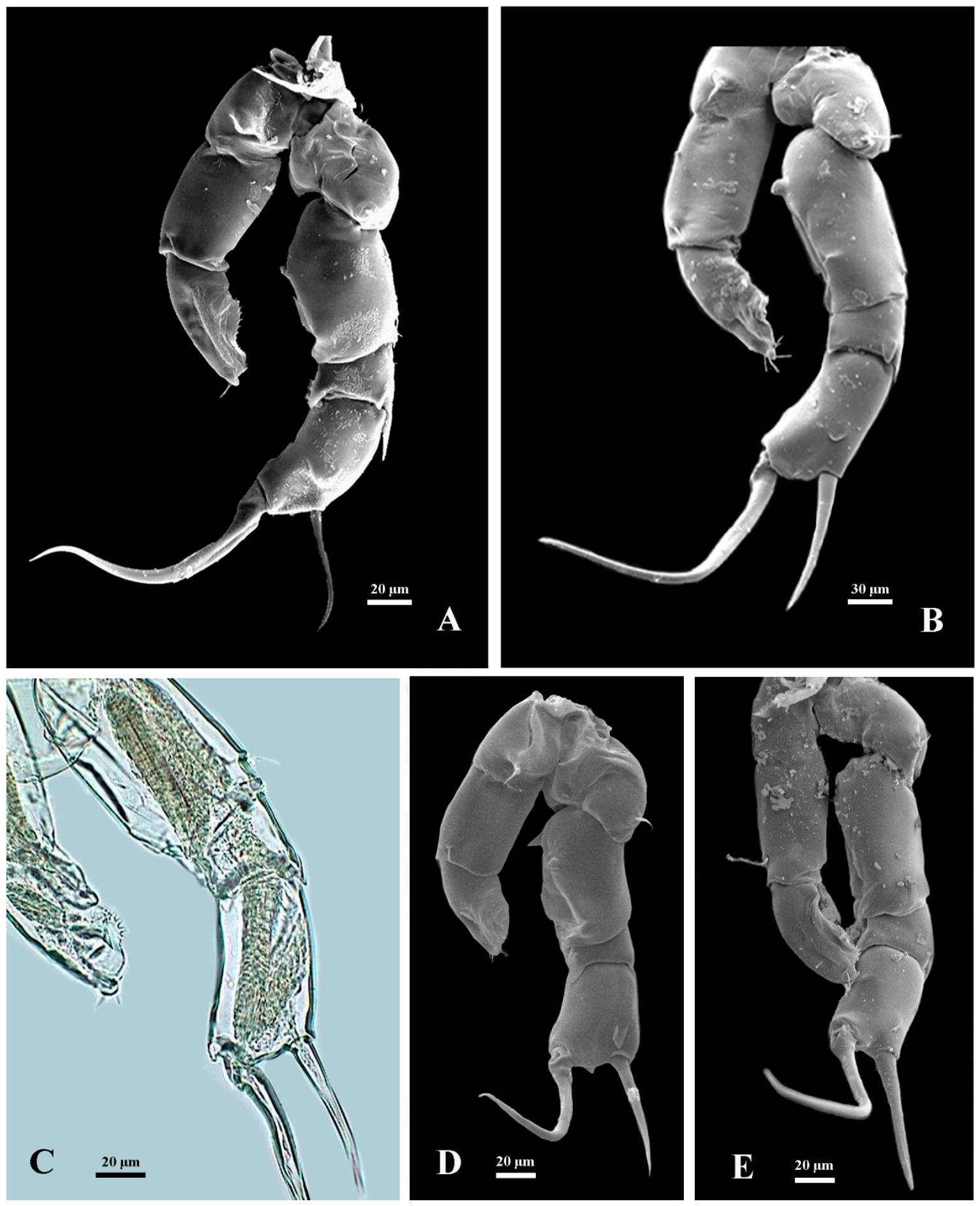

Figure 6. Photographs of the male leg 5 (P5) of four species of the genus Tropodiaptomus: (A) T. hebereri; (B) T. oryzanus; (C) T. oryzanus, showing detail of left P5; and (D) T. ruttneri; (E) T. vicinus. 


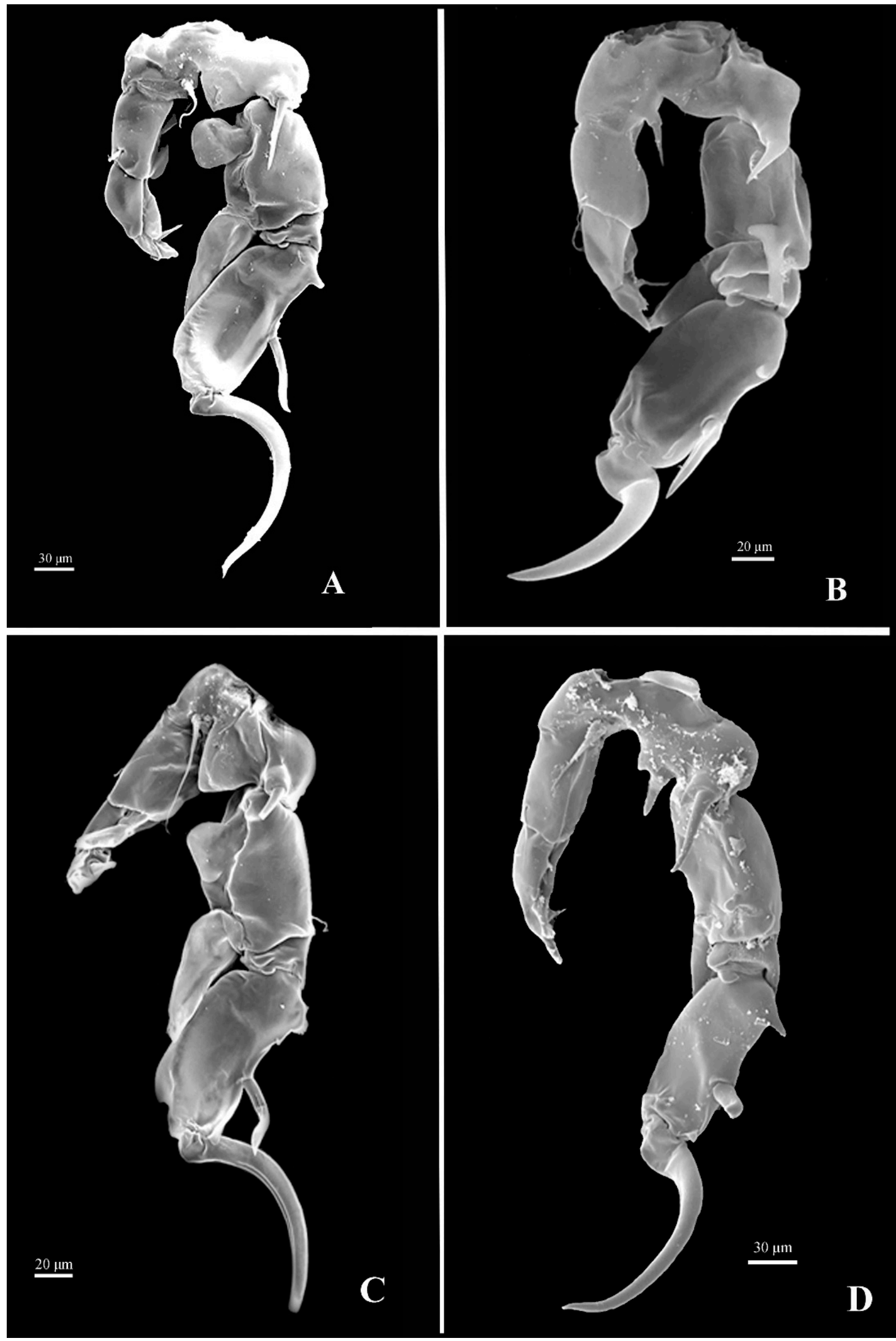

Figure 7. SEM photographs of the male leg 5 (P5) of four species of the genus Mongolodiaptomus: (A) M. botulifer; (B) M. calcarus; (C) M. malaindosinensis; and (D) M. uenoi. 

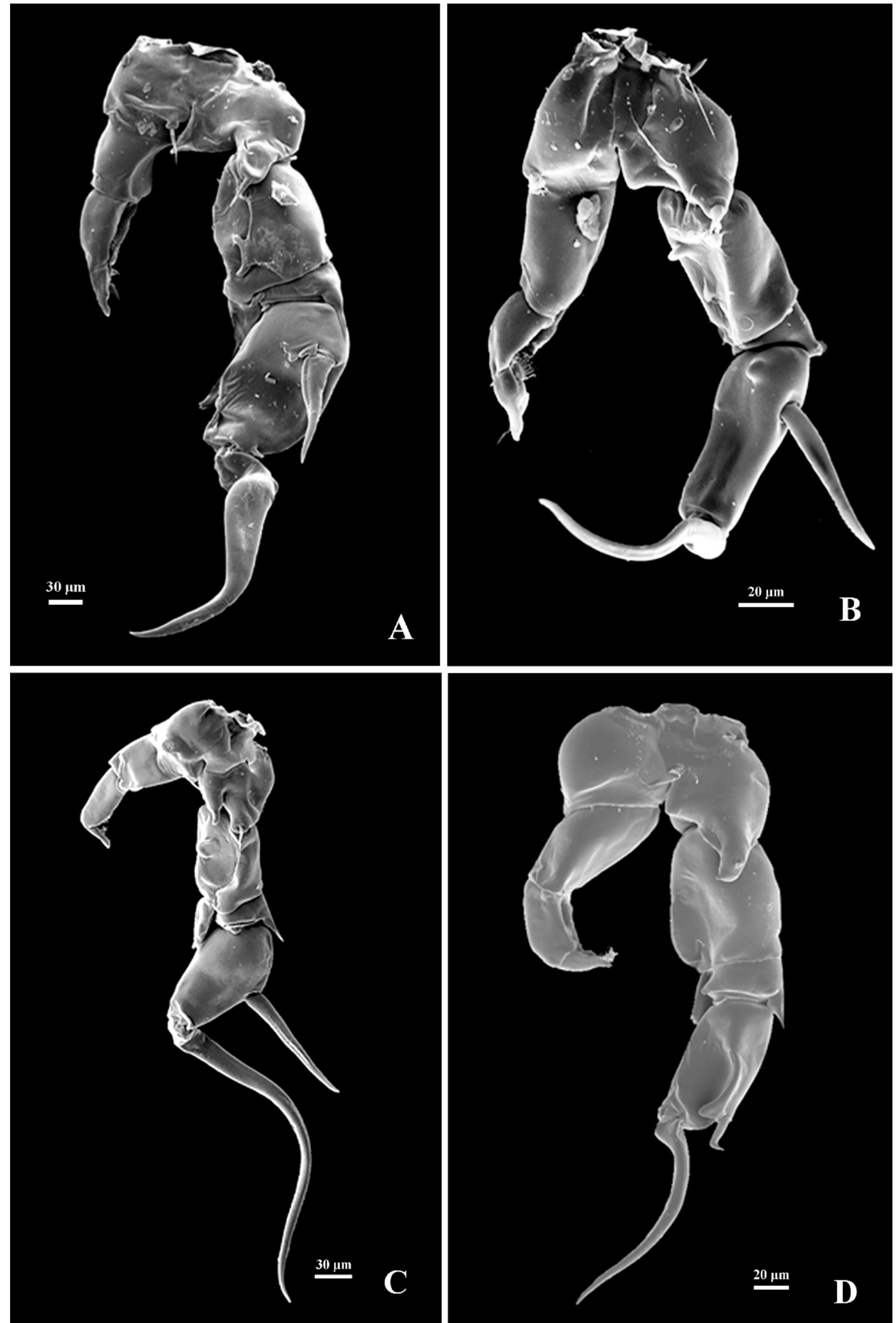

Figure 8. SEM photographs of the male leg 5 (P5) of four species of the diaptomid copepods: (A) Vietodiaptomus blachei; (B) Heliodiaptomus elegans; (C) Neodiaptomus schmackeri; and (D) Dentodiaptomus javanus. 
Table 3. Co-occurrences of up to 7 species of diaptomid copepods in freshwater habitats in Northeast Thailand.

\begin{tabular}{c}
\hline Localities \\
\hline Canal at Ban Paiklao, Chumphon Buri District, \\
Surin Province
\end{tabular}
Surin Province

2 April 1999

\section{Co-occurrence of Diaptomid Species}

1. Dentodiaptomus javanus

2. Mongolodiaptomus dumonti

3. Mongolodiaptomus malaindosinensis 4. Mongolodiaptomus uenoi

5. Neodiaptomus yangtsekiangensis

6. Phyllodiaptomus (P.) christineae

1. Dentodiaptomus javanus 2. Mongolodiaptomus dumonti 3. Neodiaptomus laii

Irrigation Canal at Ban Nayom, Sanom District, Surin Province

29 July 1999

4. Phyllodiaptomus (P.) christineae

5. Phyllodiaptomus (C.) surinensis 6. Tropodiaptomus oryzanus 7. Tropodiaptomus vicinus

1. Dentodiaptomus javanus

2. Eodiaptomus phuphanensis

Temporary pond at Ban Chaiya Buri, Chaiya Buri Sub-district, Tha Uthen District, Nakhon Phanom Province

18 May 2000

3. Heliodiaptomus phuthaiorum

4. Mongolodiaptomus malaindosinensis

5. Neodiaptomus yangtsekiangensis 6. Vietodiaptomus blachei

1. Eodiaptomus phuphanensis

Roadside canal (temporary-water habitat), KM 24, Road \#2050, Lao Suekok Sub-district, Ubon

8 June 2002

2. Mongolodiaptomus pectinidactylus Rachathani Province

3. Mongolodiaptomus uenoi

4. Neodiaptomus yangtsekiangensis

5. Tropodiaptomus oryzanus,

6. Vietodiaptomus blachei

1. Allodiaptomus raoi

2. Heliodiaptomus elegans

Lake Bueng Khong Long, Bueng Khong Long District, Bueng Kan Province

13 August 2002

3. Mongolodiaptomus pectinidactylus

4. Neodiaptomus yangtsekiangensis

5. Tropodiaptomus megahyaline

1. Eodiaptomus phuphanensis

2. Heliodiaptomus elegans

Roadside canal (temporary-water habitat), Ban Dirkai, Muangsamsip District, Ubon Rachathani

19 October 2002 Province

Temporary pond at Ban Thasongcorn, Panna Nikom District, Sakon Nakhon Province

17 May 2004

3. Mongolodiaptomus malaindosinensis

4. Neodiaptomus yangtsekiangensis

5. Phyllodiaptomus (P.) christineae 6. Vietodiaptomus blachei

1. Eodiaptomus phuphanensis

2. Mongolodiaptomus malaindosinensis

3. Mongolodiaptomus uenoi

4. Neoodiaptomus songkhramensis

5. Neodiaptomus yangtsekiangensis

6. Tropodiaptomus oryzanus,

7. Vietodiaptomus blachei

It is noted that eight species (Heliodiaptomus phuthaiorum (Figure 5A), Mongolodiaptomus loeiensis (Figure 4B), M. rarus, Neodiaptomus siamensis, N. songkhramensis, Phyllodiaptomus roietensis (Figure 2D), Tropodiaptomus sp. and Paradiaptomus greeni (Figure 5C,D)) appear to occur only in temporary waters. The northeast is the most diverse region, with 35 recorded species. The other regions have $8-17$ species.

The species recorded from Thailand can be classified according to their zoo-geographical distribution into six groups. The list of species endemic to Thailand, the lower Mekong River Basin, and Southeast Asia is provided in Table 4. Overall, 12 (28.6\%) of the species recorded appear to be endemic to Thailand. Eight species $(19 \%)$ have a more re-stricted distribution, occurring in the lower Mekong River Basin (Thailand, Laos, Cam- 
bo-dia, and Vietnam), while the other eight species are widely distributed in the countries in the lower Mekong River Basin plus the other Southeast Asian countries, including Malay-sia, Singapore, Indonesia, and the Philippines (Table 4). In addition, 10 species (23.8\%) are widely distributed in Southeast Asia and East Asia. Three species-Heliodiaptomus el-egans (Figure 8B), Heliodiaptomus viduus, and Neodiaptomus schmackeri (Figure 8C)-are the most widely distributed across Southeast, East, and South Asia. Another species with a wide distribution is Paradiaptomus greeni (Figure 5C,D), occurring in Southeast Asia, South Asia, and Africa.

\subsection{Taxonomic Checklist}

Comments on the relative occurrences, distributional information, and taxonomic status of the species recorded in the present study are provided alphabetically according to the genera. In addition, information on the geographical distribution of the species in other countries, particularly Southeast Asian countries, is documented here. Order Cal-anoida Sars, 1903; Family Diaptomidae Baird, 1850; Subfamily Diaptominae Kiefer, 1932.

\subsubsection{Genus Allodiaptomus Kiefer, 1936}

Allodiaptomus raoi Kiefer, 1936 is an uncommon species that occurrs in lakes, res-ervoirs, rivers, and rice fields in the northeast of Thailand. It was originally described in south India [37] and has been found in Cambodia [23], Vietnam [32], and China [38].

\subsubsection{Genus Arctodiaptomus Kiefer, 1932}

Arctodiaptomus sp. (Figure 5B) is identified as an undescribed species based on specimens collected from the Mun River, Ubon Ratchathani Province, northeastern Thailand. Since only one male specimen was collected, the description of this species will be provided when more specimens are available. According to the typical pincer-like processes at the end of the exopod of the male left P5 and a distinct hyaline membrane on the inner margin of the basis of both the left and right P5s in the male (Figure 5B), this species is placed in the genus Arctodiaptomus. The record of this genus in Thailand is unexpected since members of this genus inhabit water bodies across Eurasia, North Africa, and North Asia, including China [38]. In Southeast Asia, only Arctodiaptomus dorsalis (Marsh, 1907) has been recorded in more than 20 lakes in the Philippines [39]. This Neotropical species was originally described from the United States and has been found across Central Amer-ica to northern South America [40]. A. dorsalis has been introduced to the Philippines since 1991 [41]. This species has been documented to prefer eutrophic conditions. Most native calanoids may have been displaced by this invasive species.

\subsubsection{Genus Dentodiaptomus Shen and Tai, 1964}

Dentodiaptomus javanus (Grochmalicki, 1915) (Figure 8D) was originally described from Java (Indonesia) as Diaptomus javanus [42]. In 1964, this taxon was raised as a type species of the genus Dentodiaptomus by Shen and Tai (1964) [43]. The presence of at least one "denticle" or "tooth-like process" on the male left P5 Exp-2 is a unique character that was used to name the genus Dentodiaptomus [43]. Another distinct characteristic of the male right P5 of this genus is the Exp-2, a longitudinal ridge on the posterior surface that terminates near the insertion of the principal spine. This ridge is not observed in other genera of diaptomids. In Thailand, this species commonly occurs in every type of temporary habitat (rice fields, roadside canals, temporary ponds) [9] and permanent ponds or swamps. It is widely distributed in the northeastern region but sporadic in the northern, eastern, and western regions. D. javanus has been found in Cambodia [23], Indonesia [31], Vietnam [32], and southern China [38]. 
Table 4. Diaptomid copepods from Thailand are classified according to their general geographic distribution. The Lower Mekong River Basin region consists of Thailand, Myanmar, Laos, Cambodia, and Vietnam. The Southeast Asian region consists of Thailand, Myanmar, Laos, Cambodia, Vietnam, Malaysia, Singapore, the Philippines, Indonesia, and Brunei. The East Asian region consists of China, Japan, North Korea, South Korea, Mongolia, and Taiwan. The South Asian region consists of India, Sri Lanka, Afghanistan, Bangladesh, Bhutan, Nepal, and Pakistan.

\begin{tabular}{|c|c|c|c|}
\hline Distributional Regions & Number of Species & $\begin{array}{c}\% \text { of } \\
\text { Species Recorded }\end{array}$ & List of Species \\
\hline $\begin{array}{l}\text { Thailand only } \\
\text { (Endemic species) }\end{array}$ & 12 & 28.6 & $\begin{array}{c}\text { Arctodiaptomus sp., Heliodiaptomus } \\
\text { phuthaiorum, Mongolodiaptomus dumonti, M. } \\
\text { loeiensis, M. rarus, Mongolodiaptomus sp. } \\
\text { Neodiaptomus siamensis, N. songkhramensis, } \\
\text { Phyllodiaptomus (C.) surinensis, P. (P.) } \\
\text { thailandicus, Tropodiaptomus megahyaline, } \\
\text { Tropodiaptomus. sp. }\end{array}$ \\
\hline Lower Mekong River Basin & 8 & 19.0 & $\begin{array}{l}\text { Dentodiaptomus orientalis, Eodiaptomus } \\
\text { draconisignivomi, E. phuphanensis, E. } \\
\text { phuvongi, Mongolodiaptomus mekongensis, } \\
\text { Phyllodiaptomus (P.) christineae, P. (P.) } \\
\text { roietensis, Phyllodiaptomus sp. }\end{array}$ \\
\hline Southeast Asia & 8 & 19.0 & $\begin{array}{c}\text { Mongolodiaptomus botulifer, } M . \\
\text { malaindosinensis, Neodiaptomus laii, N. } \\
\text { meggitti, Phyllodiaptomus (C.) praedictus, } \\
\text { Tropodiaptomus lanaonus, T. vicinus, } \\
\text { Vietodiaptomus blachei }\end{array}$ \\
\hline Southeast Asia and East Asia & 10 & 23.8 & $\begin{array}{c}\text { Allodiaptomus raoi, Dentodiaptomus javanus, } \\
\text { Eodiaptomus sanoamuangae, } \\
\text { Mongolodiaptomus calcarus, } M . \\
\text { pectinidactylus, } M \text {. uenoi, Neodiaptomus } \\
\text { yangtsekiangensis, Tropodiaptomus hebereri, } T \text {. } \\
\text { oryzanus, T. ruttneri }\end{array}$ \\
\hline $\begin{array}{c}\text { Southeast Asia, East Asia and } \\
\text { South Asia }\end{array}$ & 3 & 7.2 & $\begin{array}{l}\text { Heliodiaptomus elegans, Heliodiaptomus } \\
\text { viduus, Neodiaptomus schmackeri }\end{array}$ \\
\hline $\begin{array}{l}\text { Southeast Asia, South Asia } \\
\text { and Africa }\end{array}$ & 1 & 2.4 & Paradiaptomus greeni \\
\hline Total & 42 & 100 & \\
\hline
\end{tabular}

Dentodiaptomus orientalis Sanoamuang and Watiroyram, 2021 is a newly described taxon, recorded from five and six localities in the floodplain of the lower Mekong River Basin in Thailand and Cambodia, respectively [44]. D. orientalis is an uncommon taxon and has been found only in the northeast of Thailand in both temporary and permanent water habitats throughout the year. The new species differs from its congener in many morphological aspects, especially on their fifth legs. The male left P5 Exp-2 of this species has three inner distal denticles instead of one as in D. javanus.

\subsubsection{Genus Eodiaptomus Kiefer, 1932}

Eodiaptomus draconisignivomi Brehm, 1952 (Figure 3D) was described from the neighborhood of the Mekong River, Cambodia, in 1952 [45]. In Thailand, it is a common species occurring in permanent water bodies such as canals, rivers, lakes, reservoirs, and swamps in the northeastern, central, eastern, and western parts of the country [10]. This species appears to be a widely distributed diaptomid in the lower Mekong River Basin, as it is known from Cambodia [46], Laos [47], and Vietnam [32].

Eodiaptomus phuphanensis Sanoamuang, 2001 (Figure 3B) is a fairly common species inhabiting both permanent and temporary habitats but has been so far reported only in the northeast [11] and western Thailand (this study). It was first collected from two res-ervoirs 
in the Phu Phan National Park in 2001 [11]. This species has been recorded in Cambodia and central Laos [47].

Eodiaptomus phuvongi Sanoamuang and Sivongxay, 2004 (Figure 3C) was described based on specimens collected from Thailand and Laos [16]. It is a fairly common species, especially in temporary habitats in northeastern Thailand, and is also found in canals, permanent ponds, and swamps. This species is morphologically most similar to E. phuphanensis by having a characteristically flat endopod on the male right P5. However, they can be distinguished from each other in the following features of the male P5: (1) the hya-line lamella on the inner margin of the right basis of E. phuvongi is semicircular, but it is rectangular in E. phuphanensis, and (2) the basis of the left P5 has a distinct spiny knob in E. phuvongi but lacks this knob in E. phuphanensis.

Eodiaptomus sanoamuangae Ranga Reddy and Dumont, 1998 (Figure 3A) was first collected from a roadside canal in Khon Kaen Province, northeastern Thailand [6]. It is a common species and has been found in every type of both permanent and temporary wa-ter bodies, except rivers [10]. This taxon was previously confused with the Chinese E. sinensis (Burckhardt, 1913) by Mashiko (1951) [48]. To date, E. sanoamuangae is known in the north, northeast, and west of Thailand (this study), and in the Yangtze River, and its tributaries in central China [48].

\subsubsection{Genus Heliodiaptomus Kiefer, 1932}

Heliodiaptomus elegans Kiefer, 1935 (Figure 8B) was briefly described based on specimens from Myanmar [49] and was redescribed from samples collected from Thailand by Ranga Reddy and Dumont (1999) [50]. It is a common species and has been found mostly in permanent water bodies, but sometimes in temporary ponds in the north, northeast, and west of Thailand. It has been reported from Cambodia [23], Bangladesh [50], Vietnam [32], and China [38].

Heliodiaptomus phuthaiorum Sanoamuang, 2004 (Figure 5A) was described from 9 of the 456 sampled localities in northeastern Thailand [13]. This species is rare and has been found only in temporary waters, such as ponds and roadside canals, in the vicinity of the Song Khram River, a tributary of the Mekong River.

Heliodiaptomus viduus (Gurney, 1916) is an uncommon species and has been recorded only in permanent water bodies (canals, lakes, swamps, ponds, and reservoirs) in the north, central, east, and west of Thailand ([9], this study). It was first described as Diaptomus viduus from Sri Lanka [51] and has been found in Myanmar and Pakistan. This species is a common diaptomid in southern India and Bangladesh [52].

\subsubsection{Genus Mongolodiaptomus Kiefer, 1937}

A criterion relating to the armature details of the second exopodal segment of the male right P5 has been proposed to differentiate the genus Mongolodiaptomus from the close congeners Neodiaptomus and Allodiaptomus by Ranga Reddy et al. (2000) [8]. Thus, some species of both genera have been transferred to Mongolodiaptomus.

Mongolodiaptomus botulifer (Kiefer, 1974) (Figure 7A) was originally described as Neodiaptomus botulifer from Malaysia [53] and redescribed using specimens from Thailand by Ranga Reddy et al. (1998) [7]. It is the most common diaptomid copepod and always occurs in great abundance in Thailand. This species inhabits every type of freshwater habitat in every region of the country. M. botulifer has also been reported in Singapore [28], Cambodia [3], Vietnam [32], and Laos [47].

Mongolodiaptomus calcarus (Shen and Tai, 1965) (Figure 7B) was originally described as Allodiaptomus calcarus from China [54] and redescribed by Ranga Reddy et al. (1998) [7]. Specimens of this species from Malaysia, Singapore, and Thailand have been confused with the Javan Neodiaptomus mephistopheles Brehm, 1933 by Lai and Fernando $(1981,1978)[34,55]$ and Schmoker et al. (2014) [28]. According to Ranga Reddy et al. (1998) [7], it is in fact M. calcarus, not N. mephistopheles. It is one of the most common diaptomids in Thai-land, occurring in every type of freshwater habitat in the north, northeast, central, west, and 
south of Thailand. M. calcarus has also been found in western Java, Indonesia, Laos [47], and Vietnam [32].

Mongolodiaptomus dumonti Sanoamuang, 2001 (Figure 4A) was discovered in a reservoir and several localities in northeastern Thailand and then described by Sanoamuang (2001) [12]. It is a common freshwater calanoid occurring in every type of freshwater hab-itat in the north, northeast, central, east, and west of Thailand.

Mongolodiaptomus loeiensis Watiroyram and Sanoamuang, 2017 (Figure 4B) was collected from a temporary pond in Loei Province of northeastern Thailand and described by Watiroyram and Sanoamuang (2017) [19]. It is an extremely rare taxon and has to date been found only in the type locality. This species was the single calanoid found in the type locality. M. loeiensis appears to be an endemic diaptomid of Thailand.

Mongolodiaptomus malaindosinensis (Lai and Fernando, 1978) (Figure 7C) was originally described as Neodiaptomus malaindosinensis based on specimens from Malaysia [56]. However, Ranga Reddy et al. (1998) [7] observed some morphological variabilities in $M$. botulifer from Thailand, leading to serious doubt about the validity of the closely allied M. malaindosinensis. Although the morphological features of the two congeners are similar, they can be distinguished from each other by the following characters: (1) male right $\mathrm{P} 5$ basis with a large, spherical hyaline lamella on the inner margin in $\mathrm{M}$. botulifer (Figure 7A) versus a triangular hyaline lamella in M. malaindosinensis (Figure 7C); (2) male right P5 Exp-2 with a blunt chitinous process on the outer proximal third margin in M. botulifer versus minute 1-4 hyaline knobs in M. malaindosinensis; (3) male right P5 Exp-2 with a narrow chitinous flange on the inner margin in M. botulifer versus the more elevated flange in M. malaindosinensis; (4) right side of female genital double-somite with an obviously botuliform out-growth (cylindrical form with rounded end or sausage-shaped) in M. botulifer versus a small, conical out-growth with a slightly dilated proximal part and gradually tapering to the tip in M. malaindosinensis. Thus, the valid status of M. malaindosinensis is confirmed. In Thailand, M. malaindosinensis is a common species occurring in lakes, permanent ponds, swamps, reservoirs, rivers, and temporary ponds and has been found throughout the country except in the west of Thailand. This species has been reported in Singapore [55] and Cambodia [46]. Recently, M. malaindosinensis has been found in two shallow meso-eutrophic and hypereutrophic lakes in Malaysia [26].

Mongolodiaptomus mekongensis Sanoamuang and Watiroyram, 2018 (Figure 4C) was discovered in freshwater ecosystems in the floodplain of the lower Mekong River Basin [20]. It is an uncommon taxon in permanent ponds and swamps but is common in tem-porary habitats such as rice fields, roadside canals, and temporary ponds in the Ubon Ratchathani Province of northeastern Thailand. This species has so far been recorded in Cambodia, Laos, and Vietnam [20].

Mongolodiaptomus pectinidactylus (Shen and Tai, 1964) (Figure 4D) was originally described as Allodiaptomus pectinidactylus from a fish pond on Hainan Island, China [43]. This species was reported for the first time in Thailand. It is a rare taxon and has been found in lakes and temporary ponds in the northeast of Thailand. This species is also found in Vietnam [32] and southern China [38].

Mongolodiaptomus rarus (Ranga Reddy, Sanoamuang and Dumont, 1998) was originally described as Allodiaptomus rarus [7]. It is an uncommon species and has been re-ported only from temporary water habitats in the northeast of Thailand.

Mongolodiaptomus uenoi (Kikuchi, 1936) (Figure 7D) was originally described as Diaptomus uenoi from Taiwan [57] and redescribed by Ranga Reddy et al. (2000) based on specimens from Thailand [8]. It is a fairly common taxon occurring in canals, swamps, reservoirs, rivers, roadside canals, and temporary ponds in the northeast of Thailand. This species has also been reported in Indonesia [8], Vietnam [32], and southern China [38].

Mongolodiaptomus $s p$. is an extremely rare, undescribed diaptomid found in the northeast of Thailand. It is morphologically similar to M. mekongensis. A detailed description of this species will be published separately. 


\subsubsection{Genus Neodiaptomus Kiefer, 1932}

Neodiaptomus laii Kiefer, 1974 was described based on specimens from Malaysia [58]. It is an uncommon species and has been found in canals, ponds, swamps, and temporary ponds in the northeast, east, and south of Thailand. This species is also found in Singapore [28] and Laos [47]. The two following diagnostic criteria of the genus Neodiaptomus are lacking: (1) the principal lateral spine is inserted generally at the middle of the outer margin of the male right P5 Exp-2 and (2) the right caudal ramus with a tooth-like chitinous structure at the inner ventro-distal corner; thus, it is more appropriate to transfer $N$. laii to the genus Heliodiaptomus.

Neodiaptomus meggitti Kiefer, 1932 was first described in Rangoon, Myanmar [59]. It was redescribed based on specimens from the South Andaman Islands, India, by Ranga Reddy (2000) [60]. N. meggitti is an extremely rare species. It was recorded for the first time in Thailand, occurring in a permanent pond in Chiang Rai Province, northern Thailand. It is also found in Malaysia [55].

Neodiaptomus schmackeri (Poppe and Richard, 1892) (Figure 8C) was originally described as Diaptomus schmackeri [61]. Among the 14 reported species of the genus Neodiaptomus [62], N. schmackeri is the most widely distributed species, being reported from southern China, the Russian Far East, Nepal, India, Bangladesh, Sri Lanka, Korea, Japan, Taiwan, the Philippines, Malaysia, Singapore [1,63] and Vietnam [32]. Recently, Alfonso et al. (2014) [64] have reported this species in eight lakes in Albania (Europe) for the first time. Since 2003, this species has most likely been introduced to Kazakhstan's Shardara Reservoir (Central Asia) with imported fish [65]. It is a fairly common species, occurring in canals, swamps, rice fields, roadside canals, and temporary ponds in the north, central and west of Thailand.

Neodiaptomus siamensis Proogkiat and Sanoamuang, 2008 was described from specimens recorded in Thailand [18]. It is an extremely rare species and has been found only in three temporary pools in Kamphaeng Phet and Phitsanulok Provinces (these provinces are sometimes designed in the northern region) in the central region of Thailand. This species has not been found in other countries.

Neodiaptomus songkhramensis Sanoamuang and Athibai, 2002 was discovered in several temporary water habitats in the vicinity of the Song Khram River, a tributary of the Mekong River [15]. It is an uncommon species, occurring in rice fields, roadside canals, and temporary ponds in northeastern Thailand. This species has not been reported in other countries.

Neodiaptomus yangtsekiangensis Mashiko, 1951 was described from samples recorded from the Yangtze River, central China [48]. It was redescribed based on specimens from Thailand by Ranga Reddy et al. (1998) [7]. N. yangtsekiangensis is a common species occurring in every type of habitat (except in rice fields) in the northeast, east, and south of Thailand. This species has been reported in southern China [38], Laos, Cambodia [46], and Vietnam [32].

\subsubsection{Genus Phyllodiaptomus Kiefer, 1936}

The genus Phyllodiaptomus Kiefer, 1936 is among the most common freshwater diaptomid copepods in Southeast Asia [9]. Members of this genus are grouped into two sub-genera, based on the distinctive structures of the male left P5 Exp-2. The subgenus Phyllo-diaptomus (Ctenodiaptomus) (Dumont, Ranga Reddy and Sanoamuang, 1996) [5] has a ser-rated hyaline fan on the inner margin of the left P5 Exp-2, while in Phyllodiaptomus (Phyl-lodiaptomus) (Dumont, Ranga Reddy and Sanoamuang, 1996), the corresponding structure bears a field of spinules.

Subgenus Phyllodiaptomus (Ctenodiaptomus)

Dumont, Ranga Reddy, and Sanoamuang, 1996

Phyllodiaptomus (Ctenodiaptomus) praedictus Dumont and Ranga Reddy, 1994 (Figure 2A) was described based on specimens from Thailand [4]. It is a common species inhab-iting every type of freshwater habitat throughout Thailand, except in the south. This spe-cies 
has also been recorded in Laos and Cambodia [46]. Another closely related subspe-cies, Phyllodiaptomus (Ctenodiaptomus) praedictus sulawesensis Alekseev and Vaillant, 2013, has been reported in Lake Tondano, North Sulawesi, Indonesia [31].

Phyllodiaptomus (Ctenodiaptomus) surinensis Sanoamuang and Yindee, 2001 was discovered in two irrigation canals in Surin Province, northeastern Thailand [14]. It is an ex-tremely rare species and has never been found again. This species appears to be an en-demic species of Thailand.

Subgenus Phyllodiaptomus (Phyllodiaptomus)

Dumont, Ranga Reddy and Sanoamuang, 1996

Phyllodiaptomus (Phyllodiaptomus) christineae Dumont, Ranga Reddy, and Sanoamuang, 1996 (Figure 2B) was described based on specimens from Thailand [5]. It is a common species and has been recorded in canals, lakes, permanent ponds, swamps, reservoirs, rivers, and temporary ponds in the north, northeast, central, east, and west of Thailand. This species has not yet been found in the south. This species has also been re-ported in Laos [46].

Phyllodiaptomus (Phyllodiaptomus) roietensis Sanoamuang and Watiroyram, 2020 (Figure 2D) was described based on specimens from Thailand and Cambodia [21]. It is a rare species, inhabiting rice fields, roadside canals, and temporary ponds only in the northeast of Thailand.

Phyllodiaptomus (Phyllodiaptomus) thailandicus Sanoamuang and Teeramaethee, 2006 (Figure 2C) was described based on specimens from Thailand [17]. It is a rare species oc-curring in permanent and temporary ponds in the northeast and the south of Thailand. This species appears to be an endemic species of Thailand.

Phyllodiaptomus (Phyllodiaptomus) sp. is a rare, undescribed diaptomid in the north-east of Thailand. It is similar to $P$. (P.) christineae. A description of this species will be pub-lished in a separate article.

\subsubsection{Genus Tropodiaptomus Kiefer, 1932}

Tropodiaptomus hebereri (Kiefer, 1930) (Figure 6A) was originally named as Diaptomus hebereri [66]. It is an extremely rare taxon that occurs in canals in the north of Thailand. This species has been found in Malaysia [67], Singapore [3], India [68], Indonesia [31], and China [38].

Tropodiaptomus lanaonus Kiefer, 1982 was described from Lake Lanao, Mindanao Island, Philippines [69]. It was recorded as an endemic diaptomid of the Philippines by Lopez et al. (2017) [30]. T. lanaonus is a rare species and has been reported from lakes in the northeast and central regions of Thailand.

Tropodiaptomus megahyaline Saetang, Sanoamuang, and Maiphae, 2020 is a newly described species from Thailand [22]. It is a rare species and has been reported from rice fields and permanent ponds in the northeast of Thailand. This species was recorded as Tropodiaptomus sp. in Lake Bueng Khong Long in Bueng Kan Province, northeastern Thailand by Teeramaethee (2006) [70].

Tropodiaptomus oryzanus Kiefer, 1937 (Figure 6B,C) was described based on samples from rice fields in Taiwan [71]. It is an uncommon species and has been found in lakes and temporary habitats such as rice fields, roadside canals, and temporary ponds in the northeast of Thailand. This species is also found in Vietnam [32], China, Korea, and Japan [38].

Tropodiaptomus ruttneri (Brehm, 1923) (Figure 6D) was originally named Diaptomus ruttneri, based on specimens from Yunnan Province, China. It is an uncommon species and has been reported from rice fields and temporary ponds in the north and northeast of Thailand. This species is also known from Malaysia [67], Japan, and Taiwan [3].

Tropodiaptomus vicinus (Kiefer, 1930) (Figure 6E) was originally described as Diaptomus vicinus based on specimens from Indonesia [72]. It is a common species and has been found in irrigation canals, lakes, permanent ponds, swamps, and rivers in the north, 
northeast, central, west, and south of Thailand. This species has been found in the Philippines [3], Malaysia [67], Cambodia [46], Indonesia [31], India [68] and Vietnam [32].

Tropodiaptomus sp. is an extremely rare, undescribed diaptomid collected from a temporary pond in the north of Thailand. Morphologically, it is similar to T. ruttneri. A description of this species will be published separately.

\subsubsection{Genus Vietodiaptomus Dang, 1977}

The genus Vietodiaptomus was created based on specimens from Vietnam, with Vietodiaptomus hatinhensis Dang, 1977 as the type species [73]. According to Walter and Box-shall (2021), the other two members of this genus are Vietodiaptomus blachei (Brehm, 1951) and Vietodiaptomus tridentatus Dang and Ho, 1985 [62]. Since the description of this genus was published in the Vietnamese language, a redescription using English is required.

Vietodiaptomus blachei (Brehm, 1951) (Figure 8A) was originally described as Eodiaptomus blachei based on specimens from Cambodia [23]. It is a common species occurring in canals, lakes, permanent ponds, swamps, reservoirs, rice fields, roadside canals, and temporary ponds, and has been recorded in the north, northeast, central, west, and south of Thailand. This species has not yet been found in the eastern region. This species has been reported from Indonesia [3], Laos [47], Malaysia [67], and Singapore [28].

Subfamily Paradiaptominae Kiefer, 1932

Genus Paradiaptomus Sars, 1895

Paradiaptomus greeni (Gurney, 1906) (Figure 5C,D) was originally described as Diaptomus greeni based on samples from Sri Lanka (Ceylon) [74]. It is also found in South Af-rica [75], Tunisia [76], India [77], and China [38]. It is an extremely rare species and has been found so far only in a temporary pond in Khon Kaen Province, northeastern Thai-land. This is the first record of a representative of the Paradiaptominae in Southeast Asia.

\section{Discussion and Conclusions}

The species diversity of freshwater diaptomid copepods (42 species) in Thailand is higher than that reported from the other Southeast Asian countries- 8 from the Philippines [30], 12 from Malaysia and Singapore [25], 17 from Indonesia [31], 19 from Laos [24], 24 from Cambodia [46], and 29 from Vietnam [32]. The comparison with other Asian countries revealed that the diaptomid diversity of Thailand is higher than Kazakhstan (26 species) [65] but comparable to that of India (about 50 species) [77] and China (56 species) [38], although the country areas of India and China are larger than Thailand by 6.4 and 18.7 times, respectively. Thus, Thailand is one of the countries with the most diversified diaptomid fauna in the world, and it is a biodiversity hotspot for diaptomid copepods in Southeast Asia.

The new record for Thailand (Paradiaptomus greeni) predominantly occurs in Africa but is also known in other Asian countries, including Sri Lanka, India [75], and the Nanning Province of southern China [38]. It is noted that we did not find two speciesTropodiaptomus doriai and Sinodiaptomus chaffanjoni-in our extensive collection. Both species were previously recorded in Thailand by Daday (1906) [34] and Boonsom (1984) [35], respectively.

Thailand has a greater record of co-occurrence (up to seven species) than India's Northern Western Ghats (up to four species) [77]. The high degree of diaptomid species co-occurrence in Thailand is remarkable, as it is uncommon to find more than one calan-oid species in a temperate lake [78]. The difference in diversity might also be partly a re-sult of artefacts due to more intensive surveying and a good basis in systematics and tax-onomy in Thailand within the region.

The species richness of diaptomid copepods (35 species) in the northeast, which is higher than in the other regions of Thailand (8-17 species), can be explained by the fact that it covers about one-third of the country and contains many temporary water bodies in 
the floodplains of major rivers, and the majority of the samples (about 60\%) were collected from this region.

In conclusion, a total of 4962 samples were analyzed from 2150 diverse freshwater habitats across Thailand, and 42 species belonging to 11 genera in 2 subfamilies of Diaptomidae were identified. The current number of species listed is the highest among those reported in other Southeast Asian countries, ranging from 8 to 29. Mongolodiaptomus is the most diverse genus with 10 species, followed by Tropodiaptomus with 7, and Neodiaptomus and Phyllodiaptomus with 6 . In total, 17 species ( $40.5 \%$ of the species recorded) have been described originally from Thailand since 1994. Eight taxa (Arctodiaptomus sp., Mongolodiaptomus pectinidactylus, Neodiaptomus meggitti, Tropodiaptomus hebereri, T. lanaonus, T. oryzanus, T. ruttneri, and Paradiaptomus greeni) are recorded for the first time in Thailand. This is the first record of a species (P. greeni) in the subfamily Paradiaptominae in Southeast Asia as well. The relative occurrences of the species recorded are categorized into five groups: 12 common, 5 fairly common, 9 uncommon, 7 rare, and 9 extremely rare species. The most frequently encountered species in the sampled localities, which are widely dis-tributed throughout the six regions of Thailand, were Mongolodiaptomus botulifer, Phyllodiaptomus praedictus, M. calcarus, M. dumonti, M. malaindosinensis, Vietodiaptomus blachei, P. christineae, Eodiaptomus sanoamuangae, Neodiaptomus yangtsekiangensis, E. draconisignivomi, Tropodiaptomus vicinus, and Heliodiaptomus elegans. The species richness of the diaptomids ranged from 1 to 7 species per locality at the same sampling dates. The coexistence of 4-7 diaptomid species has been observed in northeastern Thailand, particularly in smallsized temporary-water habitats. The high degree of diaptomid species co-occurrence in Thailand is remarkable, as it is uncommon to find more than one calanoid species in a temperate lake. Eight species appear to occur only in temporary waters. The northeast is the most diverse region, with 35 recorded species. Twelve species appear to be endemic to Thailand, and eight are endemic to the lower Mekong River Basin countries (Thailand, Cambodia, Laos, and Vietnam). This study demonstrated that species di-versity is high in temporary water habitats in the floodplains of major rivers, and Thailand is a biodiversity hotspot for diaptomid copepods in Southeast Asia. In addition, the taxonomic status of Mongolodiaptomus malaindosinensis has been confirmed.

Author Contributions: Conceptualization, L.S. and P.D.; methodology, L.S. and P.D.; formal analysis, L.S.; investigation, P.D.; resources, P.D.; data curation, L.S.; writing-original draft preparation, L.S.; writing-review and editing, L.S.; visualization, P.D.; funding acquisition, L.S. All authors have read and agreed to the published version of the manuscript.

Funding: This research was funded by a Research and Graduate Studies Grant, from Khon Kaen University, Grant Number KKU-ATRC-2564-01.

Institutional Review Board Statement: Ethical review and approval were waived for this study due to the fact that the animals that we collected from natural habitats are tiny zooplankton and microcopic in size.

Informed Consent Statement: Not applicable.

Data Availability Statement: The data presented in this study is available on request from the corresponding author.

Acknowledgments: The authors would like to thank Jittra Teeramaethee, Santi Watiroyram, Sujiphon Athibai, Kamonwan Koomput, Phuttaphannee Boonmak, Rachada Chaichareon, Supatra Tiangnga, Supatra Leckchan, Khunapat Proongkiat, Weera Yindee, Panna Wansuang, Boonthida Boonsit, Sirichai Faitakham, and Parinda Tungpanyaporn, for their assistance in the sample collections and identifications. The authors gratefully acknowledge two anonymous reviewers for their critical review of the manuscript. Nithikarn Sanoamuang and Sirikamon Phlaingam are thanked for linguistic corrections of the manuscript and figure preparation.

Conflicts of Interest: The authors declare no conflict of interest. 


\section{References}

1. Dussart, B.; Defaye, D. World Directory of Crustacea Copepoda of Inland Waters, I-Calaniformes; Backhuys Publishers: Leiden, The Netherlands, 2002; pp. 1-276.

2. Boxshall, G.A.; Defaye, D. Global diversity of copepods (Crustacea: Copepoda) in freshwater. Hydrobiologia 2008, 595, 195-207. [CrossRef]

3. Lai, H.C.; Fernando, C.H. Zoogeographical distribution of Southeast Asian freshwater Calanoida. Hydrobiologia 1980, 74, 53-66. [CrossRef]

4. Dumont, H.J.; Ranga Reddy, Y. Phyllodiaptomus praedictus n. sp. (Copepoda, Calanoida) from Thailand. Hydrobiologia 1994, 273, 101-110. [CrossRef]

5. Dumont, H.J.; Ranga Reddy, Y.; Sanoamuang, L. Description of Phyllodiaptomus christineae n. sp. from Thailand, and distinction of two subgenera within Phyllodiaptomus Kiefer, 1936 (Copepoda, Calanoida). Hydrobiologia 1996, 323, 139-148. [CrossRef]

6. Ranga Reddy, Y.; Dumont, H.J. A review of the genus Eodiaptomus Kiefer, 1932, with the description of E. sanuamuangae n. sp. from Thailand, and a redescription of E. lumholtzi (Sars, 1889) from Australia (Copepoda, Calanoida). Hydrobiologia 1998, 361, 169-189. [CrossRef]

7. Ranga Reddy, Y.; Sanoamuang, L.; Dumont, H.J. A note on the Diaptomidae of Thailand, including redescription of three species and description of a new species (Copepoda, Calanoida). Hydrobiologia 1998, 361, 201-223. [CrossRef]

8. Ranga Reddy, Y.; Sanoamuang, L.; Dumont, H.J. Amended delimitation of Mongolodiaptomus against Neodiaptomus and Allodiaptomus and redescription of the little known Mongolodiaptomus uenoi (Kikuchi, 1936) from Thailand (Copepoda: Calanoida: Diaptomidae). Hydrobiologia 2000, 418, 99-109. [CrossRef]

9. Sanoamuang, L. Species composition and distribution of freshwater Calanoida and Cyclopoida (Copepoda) of north-east Thailand In Crustaceans and Biodiversity Crisis; Schram, F.R., Klein, J.V.C., Eds.; Brill Academic Publishers: Leiden, The Netherlands, 1999; pp. 217-230.

10. Sanoamuang, L. Distributions of three Eodiaptomus species (Copepoda: Calanoida) in Thailand, with a redescription of E. draconisignivomi Brehm, 1952. Hydrobiologia 2001, 453, 565-576. [CrossRef]

11. Sanoamuang, L. Eodiaptomus phuphanensis, n. sp., a new freshwater copepod (Calanoida: Diaptomidae) from the Phu Phan National Park, Thailand. Int. Rev. Hydrobiol. 2001, 86, 219-228. [CrossRef]

12. Sanoamuang, L. Mongolodiaptomus dumonti n. sp., a new freshwater copepod (Calanoida, Diaptomidae) from Thailand. Hydrobiologia 2001, 448, 41-52. [CrossRef]

13. Sanoamuang, L. Heliodiaptomus phuthaiorum n. sp., a new freshwater copepod (Calanoida, Diaptomidae) from temporary ponds in northeast Thailand. Int. Rev. Hydrobiol. 2004, 89, 392-406. [CrossRef]

14. Sanoamuang, L.; Yindee, W. A new species of Phyllodiaptomus (Copepoda, Diaptomidae) from northeast Thailand. Crustaceana 2001, 74, 435-448.

15. Sanoamuang, L.; Athibai, S. A new species of Neodiaptomus (Copepoda, Diaptomidae) from temporary waters in northeast Thailand. Hydrobiologia 2002, 489, 71-82. [CrossRef]

16. Sanoamuang, L.; Sivongxay, N. Description of Eodiaptomus phuvongi, n. sp. (Copepoda, Calanoida) from Thailand and Laos. Crustaceana 2004, 77, 1223-1236. [CrossRef]

17. Sanoamuang, L.; Teeramaethee, J. Phyllodiaptomus thailandicus, a new freshwater copepod (Copepoda, Calanoida, Diaptomidae) from Thailand. Crustaceana 2006, 79, 475-487.

18. Proongkiat, I.; Sanoamuang, L. Description of Neodiaptomus siamensis, a new diaptomid copepod (Copepoda, Calanoida) from temporary pools in northern Thailand. Crustaceana 2008, 81, 177-189.

19. Watiroyram, S.; Sanoamuang, L. A new species of Mongolodiaptomus Kiefer, 1938 from northeast Thailand and a key to the species (Crustacea: Copepoda, Calanoida, Diaptomidae). Zookeys 2017, 710, 15-32. [CrossRef]

20. Sanoamuang, L.; Watiroyram, S. Mongolodiaptomus mekongensis, a new species of copepod (Copepoda, Calanoida, Diaptomidae) from temporary waters in the floodplain of the lower Mekong River Basin. Raffles Bull. Zool. 2018, 66, 782-796.

21. Sanoamuang, L.; Watiroyram, S. Phyllodiaptomus (Phyllodiaptomus) roietensis, a new diaptomid copepod (Copepoda: Calanoida) from temporary waters in Thailand and Cambodia, with a key to the species. ZooKeys 2020, 911, 1-20. [CrossRef]

22. Saetang, T.; Sanoamuang, L.; Maiphae, S. A new species of genus Tropodiaptomus Kiefer, 1932 (Crustacea: Copepoda: Calanoida: Diaptomidae) from Thailand. J. Nat. Hist. 2020, 54, 2297-2322. [CrossRef]

23. Brehm, V. Cladocera and Copepoda Calanoida von Cambodja. Cybium 1951, 6, 95-124.

24. Sanoamuang, L.; Watiroyram, S. Allodiaptomus nongensis, a new diaptomid copepod (Copepoda: Calanoida) from a tributary of the Mekong River, with notes on its consumption by local people in Central Laos. J. Limnol. 2019, 78, 185-200. [CrossRef]

25. Lim, R.P.; Lai, H.C. Crustacea: Copepoda, Calanoida. In Freshwater Invertebrates of the Malaysian Region; Yule, C.M., Sen, Y.H., Eds.; Academy of Sciences Malaysia: Kuala Lumpur, Malaysia, 2014; pp. 254-266.

26. Umi, W.A.D.; Yusoff, F.M.; Aris, A.Z.; Sharip, Z.; Sinev, A.Y. Planktonic microcrustacean community structure varies with trophic status and environmental variables in tropical shallow lakes in Malaysia. Diversity 2020, 12, 322. [CrossRef]

27. Alekseev, V.R.; Yusoff, F.M.; Fefilova, E.B. Continental copepod biodiversity in North-Eastern Borneo, Malaysia. Arthropoda Sel. 2016, 25, 187-197. [CrossRef]

28. Schmoker, C.; Mahjoub, M.S.; Calbet, A.; Hsiao, S.H.; Russo, F.; Larsen, O.; Trottet, A.; Drillet, G. A review of the zooplankton in Singapore waters. Raffles Bull. Zool. 2014, 62, 726-749. 
29. Mamaril, A.C. Zooplankton diversity in Philippine lakes. In Conservation and Ecological Management of Philippine Lakes in Relation to Fisheries and Aquaculture; Santiago, C.B., Cuvin-Aralar, M.L., Basiao, Z.U., Eds.; Southeast Asian Fisheries Development Center, Aquaculture Department: Iloilo, Philippines; Philippine Council for Aquatic and Marine Research and Development: Los Baños, Laguna; Bureau of Fisheries and Aquatic Resources: Quezon City, Philippines, 2001; pp. 81-93.

30. Lopez, M.L.D.; Pascual, J.A.F.; Dela Paz, E.S.P.; Rizo, E.Z.C.; Tordesillas, D.T.; Guinto, S.K.; Han, B.; Dumont, H.J.; Mamaril, A.C.; Papa, R.D.S. Annotated checklist and insular distribution of freshwater microcrustaceans (Copepoda: Calanoida \& Cyclopoida; Cladocera: Anomopoda \& Ctenopoda in the Philippines. Raffles Bull. Zool. 2017, 65, 623-654.

31. Alekseev, V.R.; Haffner, D.G.; Vaillant, J.J.; Yusoff, F.M. Cyclopoid and calanoid copepod biodiversity in Indonesia. J. Limnol. 2013, 72, 245-274. [CrossRef]

32. Tran, D.L.; Dang, N.T.; Ho, T.H. An annotated checklist of the family Diaptomidae Sars, 1903 (Copepoda, Calanoida) in Vietnam. Tap Chi Sinh Hoc. 2016, 38, 384-399. [CrossRef]

33. Bricker, K.S.; Wongrat, L.; Gannon, J.E. Composition and distribution of Crustacean plankton in twelve inland water bodies of Thailand. J. Fish. Environ. 1978, 10, 1-14.

34. Lai, H.C.; Fernando, C.H. The freshwater Calanoida (Crustacea: Copepoda) of Thailand. Hydrobiologia 1981, 76, 161-178. [CrossRef]

35. Boonsom, J. The freshwater zooplankton of Thailand (Rotifera and Crustacea). Hydrobiologia 1984, 113, 223-229. [CrossRef]

36. Wikipedia. Regions of Thailand, Wikimedia Foundation. Available online: https://en.wikipedia.org/wiki/RegionsofThailand (accessed on 26 June 2021).

37. Kiefer, F. Indische Ruderfusskrebse (Crustacea, Copepoda). Zool. Anz. 1936, 113, 136-142.

38. Li, H.; Dumont, H.J.; Han, B.P.; Lin, Q. Updated checklist and distribution of the diaptomid copepods (Copepoda, Calanoida, Diaptomidae) of China. Crustaceana 2018, 91, 335-352. [CrossRef]

39. Tordesillas, D.T.; Paredes, P.M.F.; Villaruel, K.P.E.; Queneri, C.A.A.M.; Rico, J.L.; Ban, S.; Papa, R.D.S. Effects of food concentration on the reproductive capacity of the invasive freshwater calanoid copepod Arctodiaptomus dorsalis (Marsh, 1907) in the Philippines. J. Crust. Biol. 2018, 38, 101-106. [CrossRef]

40. Reid, J.W. Arctodiaptomus dorsalis (Marsh): A case history of copepod dispersal. Banisteria 2007, 1860, 3-18.

41. Papa, R.D.S.; Li, H.; Tordesillas, D.T.; Han, B.; Dumont, H.J. Massive invasion of Arctodiaptomus dorsalis (Copepoda, Calanoida, Diaptomidae) in Philippine lakes: A threat to Asian zooplankton biodiversity? Biol. Invasions 2012, 14, 2471-2478. [CrossRef]

42. Grochmalicki, J. Beitrag zur Kenntnis der süsswassefauna Javas: Phyllopoda, Copepoda und Ostracod. Bulletin International de 1'Académie des Sciences de Cracovie, Classe des Sciences Mathématiques et Naturelles. Serie B Sci. Nat. 1915, 1915, $217-242$.

43. Shen, C.J.; Tai, A.Y. Descriptions of eight new species of freshwater copepods chiefly from the Pearl River delta, south China. Acta Zootaxo. Sin. 1964, 16, 225-239.

44. Sanoamuang, L.; Watiroyram, S. New species of copepod (Copepoda, Calanoida) from the floodplain of the lower Mekong River Basin in Thailand and Cambodia, with amended diagnosis of the genus Dentodiaptomus Shen \& Tai, 1964. Raffles Bull. Zool. 2021, in press.

45. Brehm, V. Vorläufige Mitteilung über einen neuen Eodiaptomus aus Hinterindien. Anz. Öst. Akad. Wiss. Wien. 1952, 89, $215-217$.

46. Chaicharoen, R. Diversity and Distribution of Freshwater Calanoid and Cyclopoid Copepods in 7 Provinces of Cambodia. Ph.D. Thesis, Graduate School, Khon Kaen University, Khon Kaen, Thailand, 2011; pp. 1-216.

47. Sanoamuang, L. Freshwater Zooplankton: Calanoid Copepods in Thailand; Klangnanatham Publishers: Khon Kaen, Thailand, 2002; pp. 1-159.

48. Mashiko, K. Studies of the freshwater plankton of central China. II. Sci Rep. Kanazawa Univ. 1951, 1, 137-154.

49. Kiefer, F. Beiträge zur Copepodenkunde (XVII). Zool. Anz. 1935, 109, 88-93.

50. Ranga Reddy, Y.; Dumont, H.J. Redescription of Heliodiaptomus elegans Kiefer, 1935, a rare south-east Asian calanoid copepod. Hydrobiologia 1999, 394, 145-152. [CrossRef]

51. Gurney, R. On some Freshwater Entomostraca from Ceylon. Proc. Zool. Soc. Lond. 1916, 86, 333-343. [CrossRef]

52. Ranga Reddy, Y. Copepoda: Calanoida: Diaptomidae. Key to the Genera Heliodiaptomus, Allodiaptomus, Neodiaptomus, Phyllodiaptomus, Eodiaptomus, Arctodiaptomus and Sinodiaptomus; SPB Academic: The Hague, Philippines, 1994; pp. 1-221.

53. Kiefer, F. Zwei neue Ruderfusskrebse (Crustacea, Copepoda) aus Malaysia. Beitr. Naturk. Forsch. Südwestdeutschl. 1974, 33, 219-222.

54. Shen, C.J.; Tai, A.Y. Descriptions of six new species of freshwater copepods chiefly from the Pearl River delta, South China. Acta Zool. Sin. 1965, 2, 126-140.

55. Lai, H.C.; Fernando, C.H. The freshwater Calanoida (Crustacea: Copepoda) of Singapore and peninsular Malaysia. Hydrobiologia 1978, 61, 113-127. [CrossRef]

56. Lai, H.C.; Fernando, C.H. Redescription of Neodiaptomus botulifer Kiefer and one of its related species. Hydrobiologia 1978, 59, 229-235. [CrossRef]

57. Kikuchi, K. A new species of Diaptomus from Formosa. Proc. Imp. Acad. Jpn. 1936, 12, 198-200. [CrossRef]

58. Kiefer, F. Eine neue Diaptomidenart aus Malaysia (Crustacea, Copepoda, Calanoida). Zool. Anz. 1974, 192, 420-424.

59. Kiefer, F. Zwei neue Diaptomiden (Copepoda, Calanoida) aus Indien. Zool. Anz. 1932, 100, 265-270.

60. Ranga Reddy, Y. Neodiaptomus meggitti Kiefer, 1932. A rare south-east Asian species from the Andaman Islands, India (Copepoda, Calanoida, Diaptomidae). Crustaceana 2000, 73, 257-272. [CrossRef] 
61. Poppe, S.A.; Richard, J. Description du Diaptomus schmackeri n. sp., recueilli par M. Schmacker dans le lac Tahoo (Chine). Bull. Soc. Zool. Fr. 1892, 17, 149-151.

62. Walter, T.C.; Boxshall, G. World of Copepods Database. Neodiaptomus Kiefer. 1932. Available online: http://www.marinespecies. org / copepoda / aphia.php? $\mathrm{p}=$ taxdetails\&id=347568 (accessed on 29 June 2021).

63. Ranga Reddy, Y. Neodiaptomus prateek n. sp., a new freshwater copepod from Assam, India, with critical review of the generic assignment of Neodiaptomus spp. and a note on diaptomid species richness (Calanoida: Diaptomidae). J. Crust. Biol. 2013, 33, 849-865. [CrossRef]

64. Alfonso, G.; Russo, R.; Belmonte, G. First record of the Asian diaptomid Neodiaptomus schmackeri (Poppe \& Richard, 1892) (Crustacea: Copepoda: Calanoida) in Europe. J. Limnol 2014, 73, 584-592.

65. Krupa, E.; Aubakirova, M. Checklist and distribution of Calanoida (Crustacea: Copepoda) in Kazakhstan (Central Asia). Water 2021, 13, 2015. [CrossRef]

66. Kiefer, F. Neue ruderfüßkrebse von den Sunda-Inseln. Zool. Anz. 1930, 86, 185-189.

67. Lim, R.P.; Fernando, C.H. A review of Malaysian freshwater Copepoda with notes on new records and little known species. Hydrobiologia 1985, 128, 71-89. [CrossRef]

68. Ranga Reddy, Y. Tropodiaptomus signatus Kiefer, 1982, a little-known species from Loktak Lake, Manipur State, India (Copepoda, Calanoida, Diaptomidae). Crustaceana 2013, 86, 1675-1688. [CrossRef]

69. Kiefer, F. Vergleichende Untersuchungen über Morphologie, Taxonomie und geographische Verbreitung der Arten der Gattung Tropodiaptomus Kiefer (Copepoda: Calaoida) aus asiatischen Binnengewässern. Hydrobiologia 1982, 93, 223-253. [CrossRef]

70. Teeramaethee, J. Species Diversity and Abundance of Rotifers, Cladocerans and Copepods in Two Wetlands: Bueng Boraphet, Nakhon Sawan Province and Bueng Khong Long, Nong Khai Province. Ph.D. Thesis, Graduate School, Khon Kaen University, Khon Kaen, Thailand, 2006; pp. 1-161.

71. Kiefer, F. Süsswassercopoden aus Ostasien. II. Neue Diaptomiden und Cyclopiden von der Insel Formosa. Zool. Anz. 1937, 119, 58-64.

72. Kiefer, F. Beitrage zur Copepodenkunde. 38. Zur Kenntnis des Diaptomus orientalis Brady-Sars. Zool. Anz. 1930, 87, 120-122.

73. Dang, N.T. New diaptomid crustacean from Vietnam. Tap Chi Sinh Vat-Dia Hoc. 1977, 15, 97-99, (In Vietnamese with English summary).

74. Gurney, R. On two new Entomostraca from Ceylon. Spolia Zeylan. 1906, 4, 126-134.

75. Rama Devi, C.; Ranga Reddy, Y. The complete postembryonic development of Paradiaptomus greeni (Gurney, 1906) (Copepoda, Calanoida) reared in the laboratory. Crustaceana 1989, 56, 141-161. [CrossRef]

76. Turki, S.; Turki, B. Copepoda and Branchiopoda from Tunisian temporary waters. Int. J. Biodivers. Conserv. 2010, $2,86-97$.

77. Kulkarni, M.R.; Pai, K. The freshwater diaptomid copepod fauna (Crustacea: Copepoda: Diaptomidae) of the Western Ghats of Maharashtra with notes on distribution, species richness and ecology. J. Limnol. 2016, 75, 135-143. [CrossRef]

78. Burns, C.W. New records of distribution and co-occurrence of freshwater calanoid copepods in New Zealand (Note). N. Z. J. Mar. Freshw. Res. 1987, 21, 149-150. [CrossRef] 